\title{
An Ionospheric Es Layer Clutter Model and Suppression in HF Surfacewave Radar
}

\author{
Yajun Li, Yinsheng Wei, Rongqing Xu, Zhuoqun Wang, and Tianqi Chu \\ Research Institute of Electronic Engineering Technology, Harbin Institute of Technology, No. 807, Harbin 150001, China
}

Correspondence should be addressed to Yinsheng Wei; weiys@hit.edu.cn

Received 31 March 2013; Revised 28 May 2013; Accepted 29 May 2013

Academic Editor: Krzysztof Kulpa

Copyright (C) 2013 Yajun Li et al. This is an open access article distributed under the Creative Commons Attribution License, which permits unrestricted use, distribution, and reproduction in any medium, provided the original work is properly cited.

This paper based on a fast implemented multiphase screen method using DFT puts forward an ionospheric Es layer clutter model and uses the newly developed dimensionality reduction space-time adaptive processing- (STAP-) JDL algorithm to suppress Es layer clutter, which proves the validity of the proposed model. Firstly, the multiphase screen method was analyzed, and a fast algorithm using DFT was proposed. Then, based on the multiphase screen method and thorough simulation, we reached a conclusion of the high-frequency radio wave propagation's fluctuation characteristics in the ionosphere. According to the results of the analysis, a new Es layer ionospheric clutter model was established and was compared with the measured data and verification was made. Finally, based on the built clutter model, JDL algorithm was applied to the high-frequency surface wave radar ionospheric clutter suppression, using the measured data to verify the validity of the model and algorithm. The simulation results showed that the built model can show the characteristics of the ionospheric Es layer clutter and that the JDL algorithm can suppress ionospheric Es layer clutter quite effectively.

\section{Introduction}

HF surface wave radar has been gradually applied as an efficient tool to detect the ocean dynamics elements and to maneuver targets with low speed, especially to monitor exclusive economic zone (EEZ) globally. When HF surface wave radar emits electromagnetic waves to the surface of the sea horizontally, some energy is emitted to the sky and reflected by the ionosphere owing to the nonideality of the antenna in the zenith direction, which forms the ionospheric clutter finally. Ionospheric clutter mainly locates at the height of $100 \mathrm{~km} \sim 120 \mathrm{~km}$ in the sporadic-E layer (Es layer) and above $210 \mathrm{~km}$ in the $\mathrm{F}$ layer, and due to the nonstationary property of the ionosphere, the sea echo is often completely submerged in the ionospheric clutter, which causes the radar's detection performance to decrease dramatically. Ionospheric clutter has become the bottleneck that limits the development of remote HF ground wave radar currently, and especially the Es layer clutter in it that has the ionosphere irregularities structure, which makes the suppression of the clutter more difficult [1].

At present, according to the relevant literature and the tests results, most adaptive methods that suppress ionospheric clutter are often more suitable for processing the steady or slowly varying disturbances. Because of the complex features of the ionospheric medium itself, the methods in the literature always fail. The main reason is that the problem is difficult to break through in the perspective of signal processing. Therefore, it is necessary to study the characteristics of radio wave propagation in the ionosphere and establish a reasonable ionospheric clutter model to suppress the clutter essentially.

Es layer of the ionosphere can be seen as a random medium. Some primary methods to deal with the problems of the wave propagation in the random medium include geometrical optics approximation, the Born approximation method, the Rytov approximation method, and multiphase screen method [2-6]. Geometrical optics approximation can be only applied to the situation that the wavelength of the radio waves is small enough to be neglected comparing with the characteristic dimension of the medium. The Born approximation is the single scattering solution of the scattering problem and it approximates the ionosphere propagation in the condition of weak fluctuation. However, multiphase screen method equals the ionosphere into several phase screens, taking the hierarchical characteristic of the 
ionosphere into consideration; so, we could simulate the propagation of the radio waves with high qualities simply selecting a sufficient number of phase screen in the ionosphere. Furthermore, it is suitable for the case of the weak and strong fluctuation, but its calculation speed is very slow.

Based on what is presented previously, the paper proposed an ionospheric Es layer clutter model using multiphase screen method with DFT fast implementation, and then, we applied STAP method to suppress the Es layer clutter under this model. At the beginning, we analyzed the basic principle of the multiphase screen method in the disturbed layered ionosphere and proposed a fast algorithm implemented by DFT. Then, we obtained the fluctuating characteristics of the high-frequency radio wave propagation in the ionosphere according to the experiment simulation at both weak and strong scintillation. Using the statistical analysis results of the multiphase screen method together with the height variation of the reflection points in the ionospheric Es layer, we established a new reflecting model in the ionospheric Es layer. We also compared and analyzed the model with the measured data to verify the reasonableness and correctness. Finally, based on the proposed ionospheric clutter model, we used the newly developed descending dimensional STAPJDL algorithm to suppress the Es layer clutter, and the simulation with measured data verified the ability of this algorithm to suppress the ionospheric clutter. The simulation results showed that the established model can present the characteristics of the ionospheric Es layer clutter basically, and the JDL algorithm can suppress ionospheric Es layer clutter quite effectively. The method proposed in this paper provided a theoretical basis for the ionospheric clutter suppression techniques and the use of STAP in anti-ionospheric clutter.

\section{The Analysis of Radio Wave's Propagation Characteristics in the Ionospheric Es Layer Based on the Multiphase Screen Method}

2.1. The Basic Theory of the Multiscreen Method. Multiphase screen technology equals the irregularities' path of the highfrequency radio wave signals in the ionosphere to a plurality of thin screens that only change the phase of the signal, and each thin screen corresponds to the impact of the ionospheric on radio wave signal over path, while we assumed that it was vacuum between each of the thin screens, that is, using the principle of equivalent. The propagation of high-frequency waves on the screen causes the signal phase to fluctuate, and the diffraction effect between the thin screens leads to the fluctuation of the amplitude and phase.

Assume that the electric field of the incident wave is field $E_{0}(z)$ with no irregularities, and after passing through the ionosphere its form $E(\vec{r})$ is as follows:

$$
E(\vec{r})=u(\vec{r}) E_{0}(z)
$$

where

$$
u(\vec{r})=\exp [-j \Phi(\vec{r})]
$$

To illustrate fluctuation of the amplitude and phase, respectively, set $u(\vec{r})$ as the following form $[2,3]$ :

$$
u(\vec{r})=\exp \left[\chi(\vec{r})-j S_{1}(\vec{r})\right]=\exp [-\phi(\vec{r})],
$$

where $\chi(\vec{r})$ is the amplitude part of $u(\vec{r})$ and $S_{1}(\vec{r})$ is the phase part of $u(\overrightarrow{\mathrm{r}})$. We can use Kirchhoff diffraction formula to solve the problem that radio waves propagate from one screen to another. Besides in the forward scattering assumption, the Kirchhoff diffraction can produce the results as follows:

$$
u(\vec{p}, z)=\frac{j k A_{0}}{2 \pi z} \iint e^{-j\left[\phi\left(\vec{p}^{\prime}\right)+(k / 2 \pi)\left|\vec{p}-\vec{p}^{\prime}\right|^{2}\right]} d^{2} \vec{p}^{\prime} .
$$

In the condition of shallow screen, we can get the relationship from the previous formulas:

$$
\begin{aligned}
& \chi(\vec{p}, z)=\frac{k}{2 \pi z} \iint \phi\left(\vec{p}^{\prime}\right) \cos \left[\frac{k\left|\vec{p}-\vec{p}^{\prime}\right|^{2}}{2 z}\right] d^{2} \vec{p}^{\prime}, \\
& S_{1}(\vec{p}, z)=\frac{k}{2 \pi z} \iint \phi\left(\vec{p}^{\prime}\right) \sin \left[\frac{k\left|\vec{p}-\vec{p}^{\prime}\right|^{2}}{2 z}\right] d^{2} \vec{p}^{\prime} .
\end{aligned}
$$

The statistical characteristics of the waves through each phase screen can be obtained by calculating the moments of formula (5). Among the moments, the mean value of the amplitude and phase fluctuation is 0 , and the correlation function of the amplitude and phase fluctuations are

$$
\begin{aligned}
& B_{\chi}(\vec{p})=\iint \Phi_{\phi}\left(\vec{k}_{\perp}\right) \sin ^{2}\left(\frac{k_{\perp}^{2} z}{2 k}\right) \cos \vec{k}_{\perp} \cdot \vec{p} d^{2} k_{\perp}, \\
& B_{S_{1}}(\vec{p})=\iint \Phi_{\phi}\left(\vec{k}_{\perp}\right) \cos ^{2}\left(\frac{k_{\perp}^{2} z}{2 k}\right) \cos \vec{k}_{\perp} \cdot \vec{p} d^{2} k_{\perp},
\end{aligned}
$$

where $\Phi_{\phi_{n-1, n}}$ is power spectrum of the phase screen at $z=$ $\left(z_{n-1}+z_{n}\right) / 2$. Taking the previous formulas (6) for Fourier transform, we can have the power spectrum of the amplitude and phase fluctuations. Consider the following:

$$
\begin{aligned}
& \Phi_{\chi_{n-1, n}}\left(\vec{k}_{\perp}\right)=\sin ^{2}\left(\frac{k_{\perp}^{2} z}{2 k}\right) \Phi_{\phi_{n-1, n}}\left(\vec{k}_{\perp}\right), \\
& \Phi_{S_{n-1, n}}\left(\vec{k}_{\perp}\right)=\cos ^{2}\left(\frac{k_{\perp}^{2} z}{2 k}\right) \Phi_{\phi_{n-1, n}}\left(\vec{k}_{\perp}\right) .
\end{aligned}
$$

In order to simplify the discussion and calculation of the problem, we only consider the two-dimensional case which assumes that the irregularities are independent of $y$ direction; thus, the relationships in the previous formulas become

$$
\begin{aligned}
& \Phi_{\chi_{n-1, n}}\left(k_{x}\right)=\sin ^{2}\left(\frac{k_{x} z}{2 k}\right) \Phi_{\phi_{n-1, n}}\left(k_{x}\right), \\
& \Phi_{S_{n-1, n}}\left(k_{x}\right)=\cos ^{2}\left(\frac{k_{x} z}{2 k}\right) \Phi_{\phi_{n-1, n}}\left(k_{x}\right) .
\end{aligned}
$$

Ionosphere can be characterized by the relative dielectric constant $\varepsilon(\vec{r})$, which is composed of regular portion 
$\langle\varepsilon(z)\rangle(z>0)$ and the random part $\varepsilon_{1}(\vec{r})$. In the disturbed stratified ionosphere, the relative dielectric constant can be expressed as

$$
\begin{gathered}
\varepsilon(x, z)=\langle\varepsilon(z)\rangle+\varepsilon_{1}(x, z), \\
\langle\varepsilon(z)\rangle=1-\frac{e^{2}}{m \varepsilon_{0} \omega^{2}}\langle N(z)\rangle=1-\frac{z}{z_{\Gamma}}, \\
\varepsilon_{1}(x, z)=\frac{-e^{2}}{m \varepsilon_{0} \omega^{2}} \Delta N(x, z)=-\left(\frac{z}{z_{\Gamma}}\right) N_{f}(x, z),
\end{gathered}
$$

where $e$ and $m$ are the charge and mass of the electron; $\langle N(z)\rangle$ is the electron concentration of the background, and it is a linear function of $z ; \Delta\langle N(x, z)\rangle$ is its fluctuations portion. $N_{f}(x, z)$ is the relative fluctuation of the electron concentration:

$$
N_{f}(x, z)=\frac{\Delta N(x, z)}{\langle N(z)\rangle} .
$$

The power spectrum of the ionospheric electron concentration fluctuation of the two-dimensional irregularities can be characterized using the available Shkarofsky spectrum:

$$
\begin{aligned}
\Phi_{N_{f}}\left(k_{x}, k_{z}\right)= & \frac{\sigma_{N_{f}}^{2}\left(k_{0} r_{0}\right)^{(p-3) / 2} r_{0}^{3} K_{p / 2}\left(r_{0} \sqrt{k_{x}^{2}+k_{z}^{2}+k_{0}^{2}}\right)}{(2 \pi)^{3 / 2} K_{(p-3) / 2}\left(k_{0} r_{0}\right)} \\
& \cdot\left(r_{0} \sqrt{k_{x}^{2}+k_{z}^{2}+k_{0}^{2}}\right)^{-p / 2},
\end{aligned}
$$

where $r_{0}$ and $L_{0}=2 \pi / k_{0}$ are the internal and external dimensions of the irregularities, $K_{v}$ is the modified Bessel function of the second kind with the order $v$. By solving the Helmholtz equation of radio wave propagation, we can yield

$$
u\left(x, z_{n}\right)=u\left(x, z_{n-1}\right) e^{j \phi_{n-1, n}(x)}
$$

where

$$
\phi_{n-1, n}(x)=-\frac{k}{2} \int_{z_{n-1}}^{z_{n}} \frac{\varepsilon_{1}(x, z)}{\sqrt{1-z / z_{\Gamma}}} d z .
$$

Substitute formula (10) into (14) and calculate the correlation function of the phase deviation. The result is shown as follows:

$$
\begin{aligned}
B_{\phi_{n-1, n}}(x)= & \frac{k^{2}}{4}\left[z_{\Gamma} \ln \left(\frac{z_{\Gamma}-z_{n-1}}{z_{\Gamma}-z_{n}}\right)-\frac{z_{n}^{2}-z_{n-1}^{2}}{2 z_{\Gamma}}-\Delta z\right] \\
& \cdot \int_{-\infty}^{+\infty} B_{N_{f}}(x, \xi) d \xi .
\end{aligned}
$$

Taking formula (15) for FFT, we can get the power spectrum of the phase deviation:

$$
\begin{aligned}
\Phi_{\phi_{n-1, n}}\left(k_{x}\right)= & \frac{\pi k_{x}^{2}}{2}\left[z_{\Gamma} \ln \left(\frac{z_{\Gamma}-z_{n-1}}{z_{\Gamma}-z_{n}}\right)-\frac{z_{n}^{2}-z_{n-1}^{2}}{2 z_{\Gamma}}-\Delta z\right] \\
& \left.\cdot \Phi_{N_{f}}\left(k_{x}, k_{z}\right)\right|_{k_{z}=0} \\
= & \frac{\pi k_{x}^{2}}{2}\left[z_{\Gamma} \ln \left(\frac{z_{\Gamma}-z_{n-1}}{z_{\Gamma}-z_{n}}\right)-\frac{z_{n}^{2}-z_{n-1}^{2}}{2 z_{\Gamma}}-\Delta z\right] \\
& \cdot \Phi_{N_{f}}\left(k_{x}, 0\right) .
\end{aligned}
$$

Formula (16) links up the power spectrum of the phase deviation and the power spectrum of the undulating electron concentration.

According to the previously obtained power spectra of the phase deviation in the phase screen, we substitute it into formula (8a) and (8b) to obtain the corresponding power spectrum of the amplitude and the phase fluctuations. As long as the power spectrum of the amplitude and phase fluctuations are obtained, the amplitude and phase deviation of the simulation phase screen in the $z=\left(z_{n-1}+z_{n}\right) / 2$ can be obtained via the numerical procedure using FFT. Assume that the horizontal size of each phase screen is $L_{h}$ and that it is divided into $N$ equal parts, the fluctuations of the random amplitude and phase at the point $x=m \cdot \Delta x$ can be described as

$$
\begin{aligned}
& \chi_{n-1, n}(m \cdot \Delta x)=\sum_{s=0}^{N-1} \sqrt{\Phi_{\chi_{n-1, n}}(s \cdot \Delta k) \cdot \Delta k} \cdot \cos \left(\frac{2 \pi s m}{N}+\varphi_{s}\right), \\
& S_{n-1, n}(m \cdot \Delta x)=\sum_{s=0}^{N-1} \sqrt{\Phi_{S_{n-1, n}}(s \cdot \Delta k) \cdot \Delta k} \cdot \cos \left(\frac{2 \pi s m}{N}+\varphi_{s}\right),
\end{aligned}
$$

where $m=0,1,2, \ldots, N-1 ; \Delta x=L_{h} / N ; \Delta k=2 \pi / L_{h}$; here, we also introduce a random phase angle $\varphi_{s}$, which is subjected to the uniform distribution in the interval $0 \sim 2 \pi$ and meets the following qualifications: $\varphi_{s}=-\varphi_{-s}$.

2.2. A New DFT-Based Fast Algorithms. Through the multiphase screen method previously mentioned, we obtained the expression of the signal amplitude and phase fluctuation that occur in the ionosphere. The formula is shown by summing, which will be very slow and inefficient when calculating directly. We make appropriate transform after which the calculation can be implemented by FFT algorithm. By the transformation formula of the trigonometric function, we can obtain

$$
\begin{aligned}
\cos \left(\frac{2 \pi s m}{N}+\varphi_{s}\right)= & \cos \left(\frac{2 \pi s m}{N}\right) \cos \left(\varphi_{s}\right) \\
& -\sin \left(\frac{2 \pi s m}{N}\right) \sin \left(\varphi_{s}\right)
\end{aligned}
$$


To be convenient, we can set

$$
\begin{aligned}
& x_{n-1, n}(s)=\sqrt{\Phi_{\chi_{n-1, n}}(s \cdot \Delta k) \cdot \Delta k}, \\
& x 1_{n-1, n}(s)=x_{n-1, n}(s) \cos \left(\varphi_{s}\right), \\
& x 2_{n-1, n}(s)=x_{n-1, n}(s) \sin \left(\varphi_{s}\right), \\
& y_{n-1, n}(s)=\sqrt{\Phi_{S_{n-1, n}}(s \cdot \Delta k) \cdot \Delta k}, \\
& y 1_{n-1, n}(s)=y_{n-1, n}(s) \cos \left(\varphi_{s}\right), \\
& y 2_{n-1, n}(s)=y_{n-1, n}(s) \sin \left(\varphi_{s}\right) .
\end{aligned}
$$
follows:

Then, the first item of formula (17) can be rewritten as

$$
\begin{aligned}
\chi_{n-1, n} & (m \cdot \Delta x) \\
= & \sum_{s=0}^{N-1} \sqrt{\Phi_{\chi_{n-1, n}}(s \cdot \Delta k) \cdot \Delta k} \cdot \cos \left(\frac{2 \pi s m}{N}+\varphi_{s}\right) \\
= & \sum_{s=0}^{N-1} x_{n-1, n}(s) \cdot\left(\cos \left(\frac{2 \pi s m}{N}\right) \cos \left(\varphi_{s}\right)\right. \\
& \left.-\sin \left(\frac{2 \pi s m}{N}\right) \sin \left(\varphi_{s}\right)\right) \\
= & \sum_{s=0}^{N-1} x 1_{n-1, n}(s) \cos \left(\frac{2 \pi s m}{N}\right) \\
& -x 2_{n-1, n}(s) \sin \left(\frac{2 \pi s m}{N}\right) \\
= & \frac{1}{2} \sum_{s=0}^{N-1}\left\{x 1_{n-1, n}(s)\left(\exp \left(\frac{j 2 \pi s m}{N}\right)+\exp \left(-\frac{j 2 \pi s m}{N}\right)\right)\right. \\
+j x 2_{n-1, n}(s)\left(\exp \left(\frac{j 2 \pi s m}{N}\right)\right. & \left.\left.-\exp \left(-\frac{j 2 \pi s m}{N}\right)\right)\right\}
\end{aligned}
$$

If we set $W_{N}=\exp (-j 2 \pi / N)$, then the previous formula can sequentially be simplified to the following form:

$$
\begin{aligned}
\chi_{n-1, n}(m \cdot \Delta x)= & \frac{1}{2} \sum_{s=0}^{N-1}\left\{x 1_{n-1, n}(s)\left(W_{N}^{-s m}+W_{N}^{s m}\right)\right. \\
& \left.+j x 2_{n-1, n}(s)\left(W_{N}^{-s m}-W_{N}^{s m}\right)\right\} \\
= & \frac{1}{2}\left\{\operatorname{DFT}\left(x 1_{n-1, n}\right)+N \cdot \operatorname{IDFT}\left(x 1_{n-1, n}\right)\right\} \\
& +\frac{j}{2}\left\{N \cdot \operatorname{IDFT}\left(x 2_{n-1, n}\right)-\operatorname{DFT}\left(x 2_{n-1, n}\right)\right\} .
\end{aligned}
$$

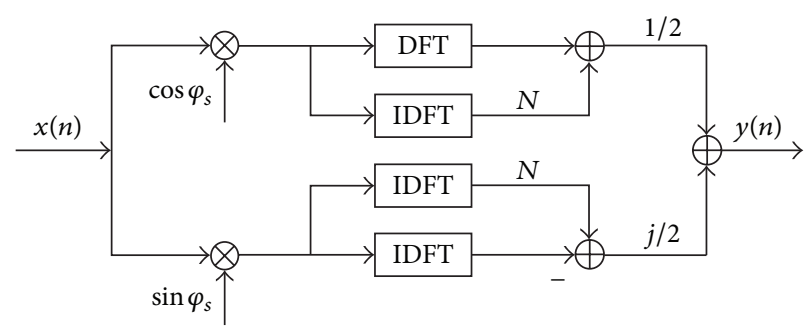

FIGURE 1: The realization of the phase screen method using DFT.

The fast algorithm for phase fluctuation can be obtained through the same method:

$$
\begin{aligned}
S_{n-1, n}(m \cdot \Delta x)= & \frac{1}{2}\left\{\operatorname{DFT}\left(y 1_{n-1, n}\right)+N \cdot \operatorname{IDFT}\left(y 1_{n-1, n}\right)\right\} \\
& +\frac{j}{2}\left\{N \cdot \operatorname{IDFT}\left(y 2_{n-1, n}\right)-\operatorname{DFT}\left(y 2_{n-1, n}\right)\right\} .
\end{aligned}
$$

We simplify the random amplitude and phase fluctuations formulas, expressing them using DFT and IDFT algorithm, respectively, which significantly improve the computational efficiency.

The multiphase screen simulation algorithm is shown as following steps.

Step 1. Substitute the corresponding parameters in the upper section into formula (12) to obtain the power spectrum of the electron concentration fluctuations.

Step 2. The obtained value of the electron concentration fluctuations would be substituted into formula (16) to get the power spectrum of the complex phase deviation.

Step 3. According to the formula (8a) and (8b), we can obtain the power spectrum of amplitude and phase fluctuations.

Step 4. Use the relation of (17) to obtain the amplitude and phase fluctuation which we can regard as a complex phase $\varphi_{n-1, n}$.

Step 5. Use formula (13) to associate the phase deviations between different phase screens with each other.

The diagram of the fast algorithm in the fourth step using DFT to implement its function is shown in Figure 1.

In Figure 1, when the input is the power spectrum of the amplitude fluctuation in the phase screen, the corresponding output is the fluctuation of the amplitude; when the input is the power spectrum of the phase fluctuation in the phase screen, the corresponding output is the fluctuation of the phase.

2.3. Simulations and Experimental Results of Multiphase Screen Method. When we use the multiphase screen method to simulate the ionospheric wave propagation problem, some values of certain parameters in the simulation process are set to be known, and the simulation results of the phase 

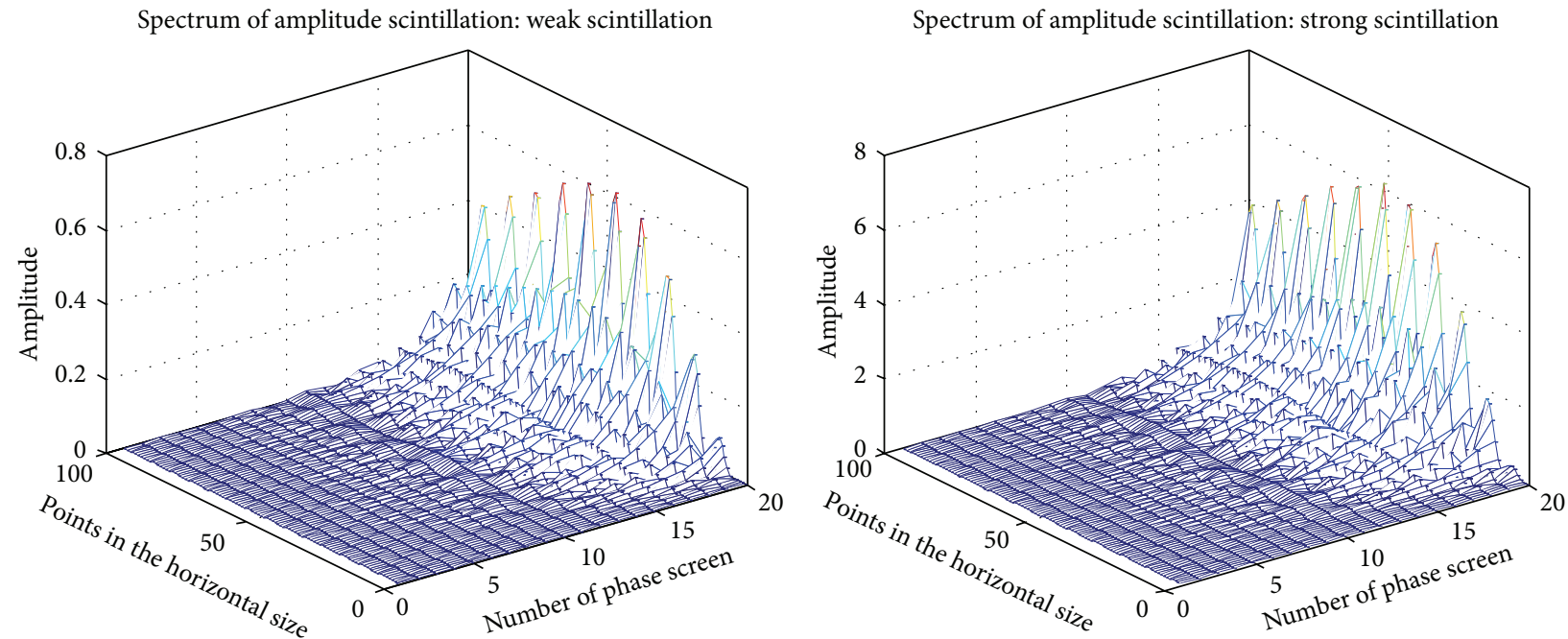

FIGURE 2: The power spectrum of amplitude scintillation.
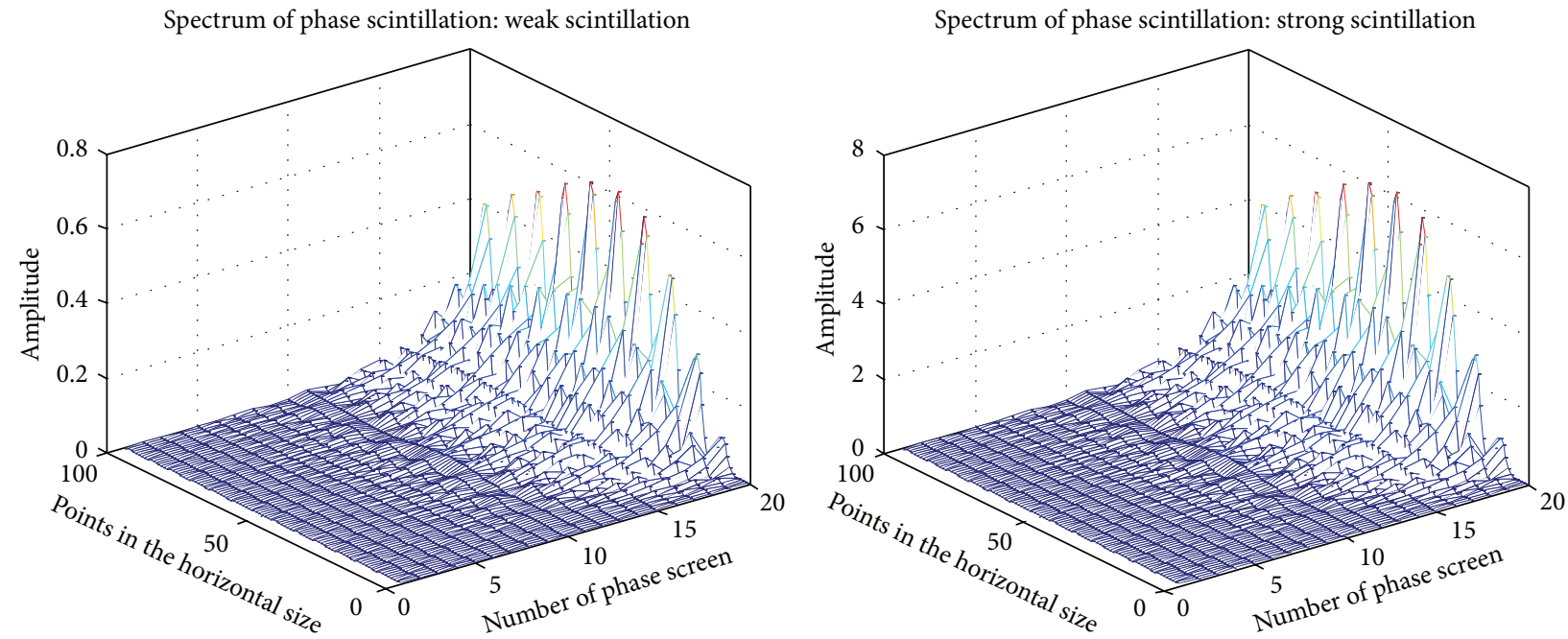

FIGURE 3: The power spectrum of phase scintillation.
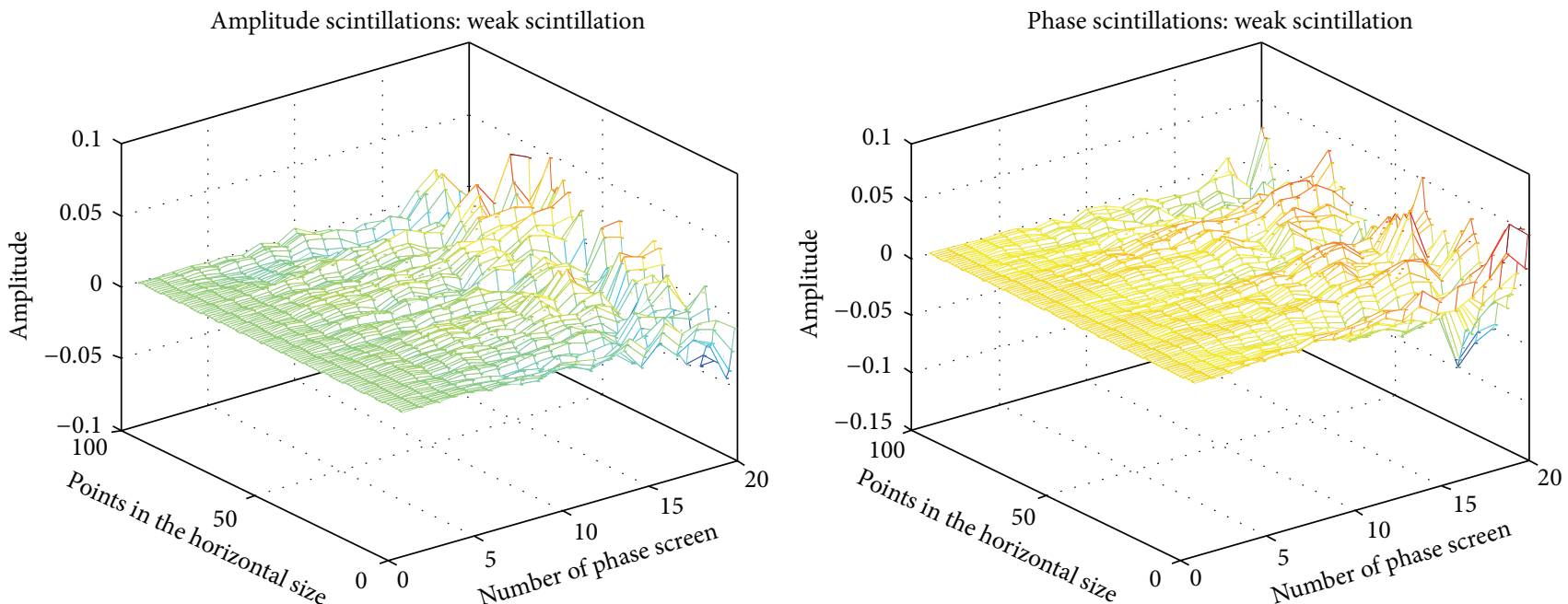

FIGURE 4: Amplitude and phase changes in phase screens in weak scintillation. 

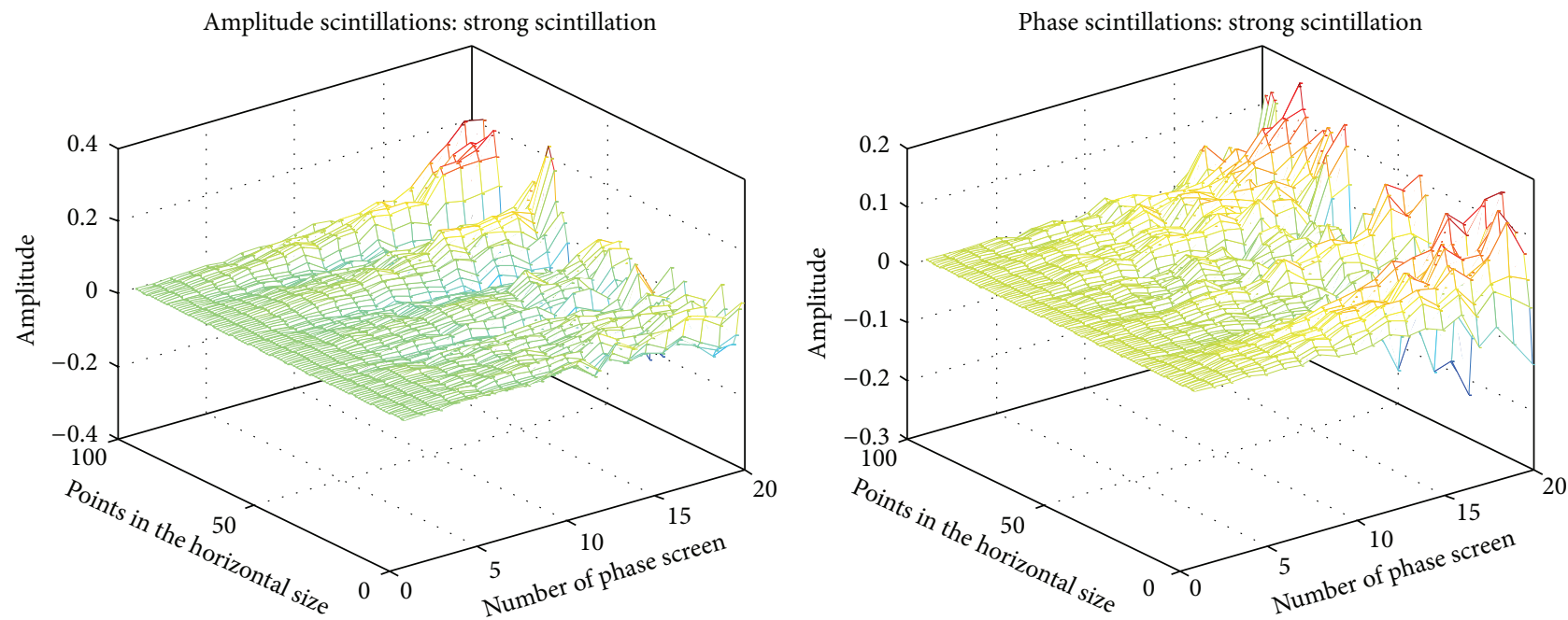

FIGURE 5: Amplitude and phase changes in phase screens in strong scintillation.

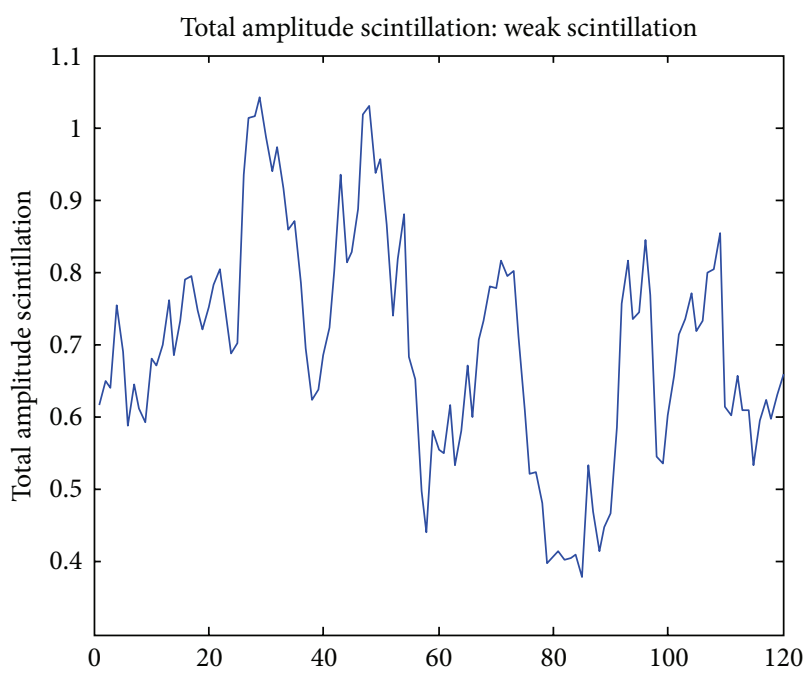

(a) Points in the horizontal size

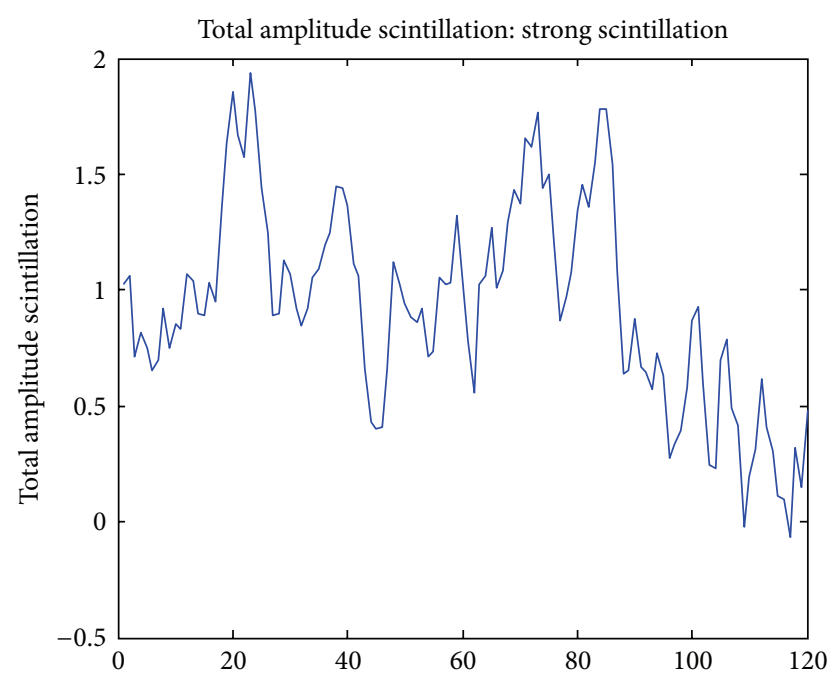

(c) Points in the horizontal size

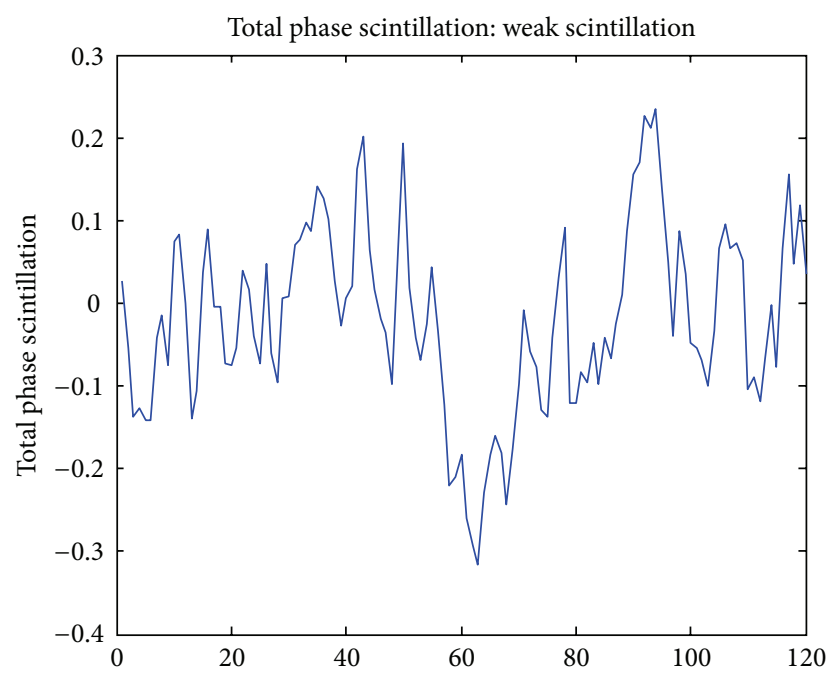

(b) Points in the horizontal size

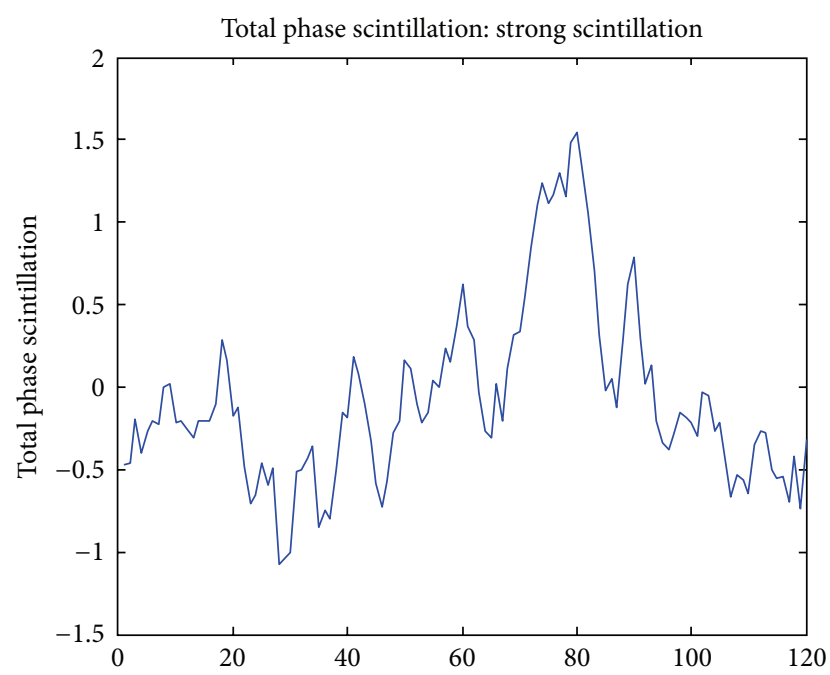

(d) Points in the horizontal size

FIGURE 6: The overall amplitude and phase scintillation. 


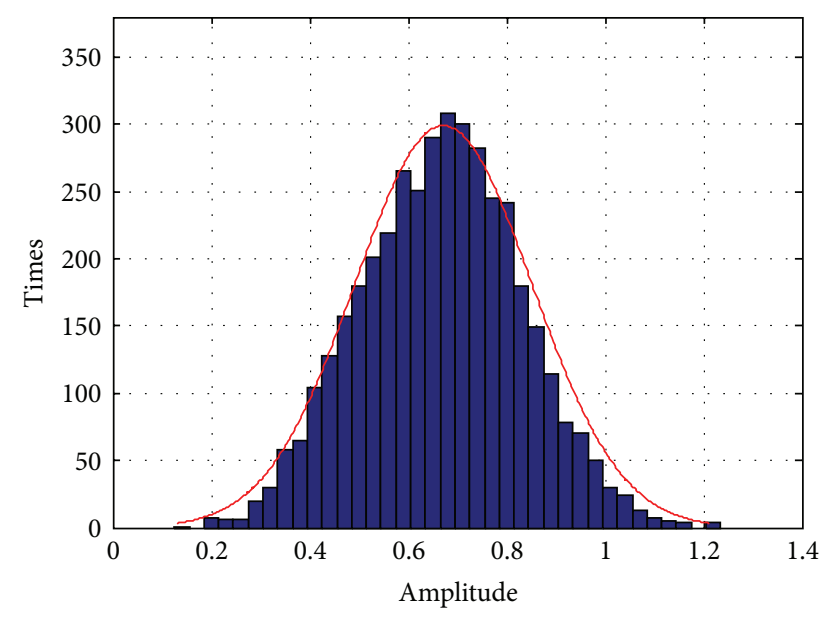

$\square$ Amplitude scintillation histogram

— Normal distribution probability density

FIGURE 7: The histogram of amplitude scintillation and fitting results of normal distribution.

screen method can be derived by using such values as initial condition. These parameters include the radar operating frequency $f=4 \mathrm{MHz}$; analog of the background medium: $Z_{\Gamma}=20 \mathrm{~km}$ (corresponding to the ionization layer region thickness when $f=4 \mathrm{MHz}$ ), $Z_{L}=90 \mathrm{~km}$ is the distance from the ground to the bottom of the ionosphere. To ensure the sampling rate and numerical calculations convenient under the simulated conditions of phase screen $[2,3]$, take a typical case. We place 20 phase screens in the ionsphere, that is, $M=20$, and thus, the relative thickness of the screen $\Delta z=$ $Z_{\Gamma} / M$; in the ionospheric $2 \mathrm{D}$ random field, electron concentration's relative fluctuation $N_{f}(r)$ was characterized by the Shkarofsky spectrum, in which the internal dimensions of the irregularities take typical value $r_{0}=15 \mathrm{~m}$, and the external dimensions take $L_{0}=2.5 \mathrm{~km}$. The spectral index of the ionospheric irregularities (i.e., the exponent) $p$ takes the value 4 or 5 and 4 herein; the horizontal dimension of each phase screen is $L_{h}=122880 \mathrm{~m}$ and is divided into $N=$ 4096 copies; the fluctuation of the electron concentration take $\sigma_{N f}=10^{-3}$ in the strong situation and $\sigma_{N f}=10^{-4}$ in the weak one. $\sigma_{N f}$ is standard deviation, indicating the strength of the turbulence of ionosphere.

2.3.1. The Power Spectrum of the Amplitude and Phase Scintillation. The power spectrum of the amplitude and phase fluctuations by complex phase screen's power spectrum using (8a) and (8b) are shown in Figures 2 and 3 in the conditions of weak and strong fluctuation.

2.3.2. The Scintillations of Amplitude and Phase. According to the derivation of the foregoing formula (21) and (22), we can obtain the amplitude and phase fluctuation value in each phase screen using the FFT algorithm, and the values are shown in Figures 4 and 5.

From Figures 4 and 5, we can see that the fluctuation of amplitude and phase is gradually increasing from the 1st to 20th phase screens because the electron concentration increases with the rising of height and the corresponding fluctuation also increases. The fluctuation of amplitude and phase at some point reaches the maximum within the same phase screen, which embodies the randomness of the ionospheric undulation in the same height. Meanwhile, in the condition of intensity fluctuation, the fluctuation of the signal amplitude and phase increases more significantly than that in the case of weak scintillation.

2.3.3. The Total Scintillation of the Amplitude and Phase. The scintillation of the amplitude and phase within each phase screen at a corresponding point is superimposed to get the overall scintillation caused by the signal going through the ionospheric region, as shown in Figure 6.

Figures 6(a) and 6(b) show the total amplitude and phase scintillation value generated by the signal going through the ionosphere in the conditions of weak scintillation, in which the fluctuation of the signal amplitude ranges from about 0.4 to 1.1 and basically concentrates on the 0.7 or 0.8 , and the fluctuation of the phase basically centre on 0 and maximizes to 0.4 radians. Figures $6(\mathrm{c})$ and $6(\mathrm{~d})$ show the total amplitude and phase fluctuation values produced by the signal going through the ionosphere in the conditions of intensity fluctuation, in which the fluctuation is significant. The amplitude's fluctuation sets 0.4 as the center basically, while the maximum fluctuation value can reach about 1.8 . The fluctuations of phase increased more than that in the case of weak fluctuation and the maximal fluctuation, can reach up to more than $1 \mathrm{rad}$.

\subsection{The Statistical Properties of the Magnitude and Phase Scintillation}

2.4.1. The Probability Distribution of the Amplitude Scintillation. Figure 7 shows the histogram of the amplitude value frequency after the signal going through ionosphere in the weak scintillation conditions using multiphase screen method, and the probability density function curve of Gaussian distribution with the parameters $(0.7,0.17)$. As we can see from Figure 7, the amplitude of the signal in the case of weak scintillation approximates the Gaussian distribution. Figure 8(a) with the q-q figure is to test whether experimental data and the given parameters' normal distribution come from the same kind of distribution. If the data in the $\mathrm{q}-\mathrm{q}$ figure approximately has a linear relationship, we can hold the opinion that the data is subjected to the Gaussian distribution. Conversely, if the data point bends seriously, they are not the same kind of distribution. From Figure 8(a), we can know that in the weak scintillation case, the fluctuation of the signal amplitude approximately obeys $\mu=0.7, \sigma_{2}=0.17$ Gaussian distribution. Figure $8(\mathrm{~b})$ is the normal probability picture (NPP) to test normal distribution. Each value in the data corresponds to a "+" sign, whose location is decided jointly by the point value and the empirical probability, and solid lines connect the $25 \%$ and $75 \%$ percentile and represent the robustness of the linear fit. The ratio of $y$ axis in the normal probability plot is uneven, and it indicates the probability 


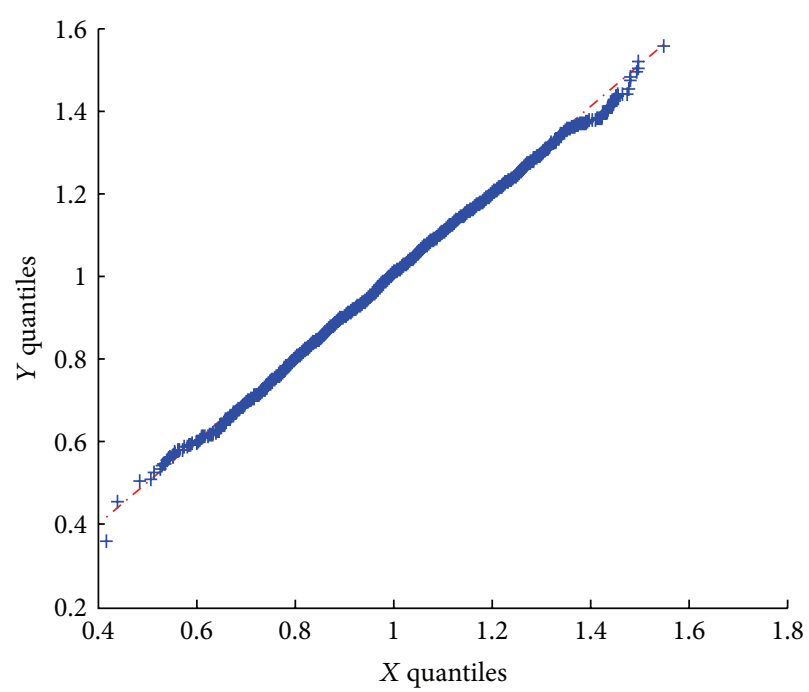

(a) Inspection in the q-q figure

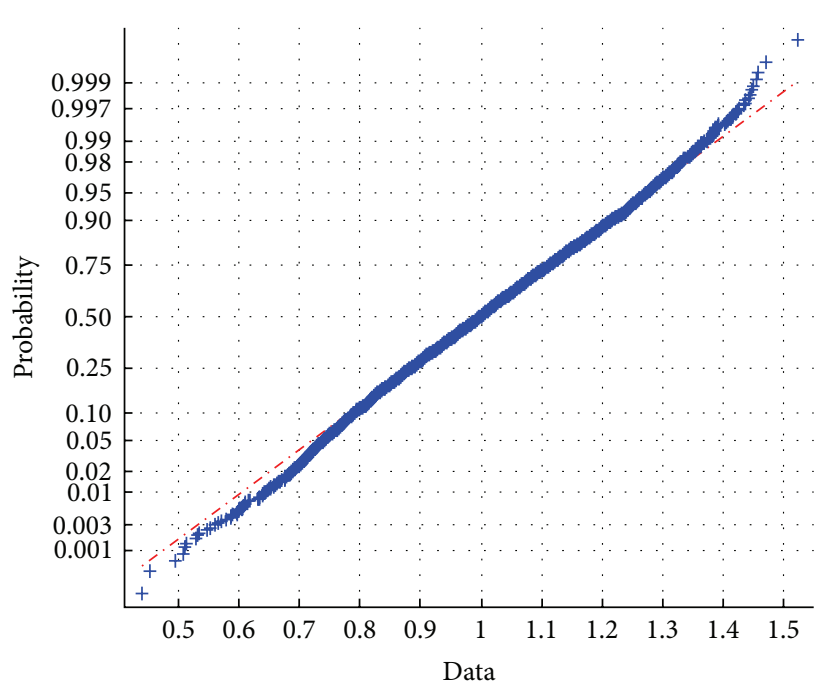

(b) Inspection in the NNP

FIGURE 8: The inspection of amplitude distribution.

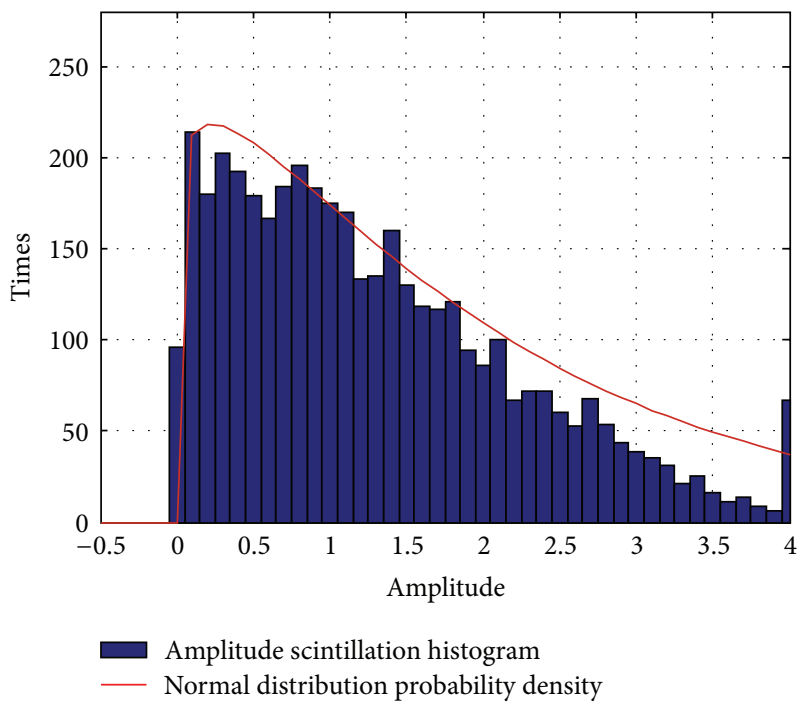

FIGURE 9: The histogram of amplitude scintillation and the fitting results of the Weibull distribution.

value between $0 \sim 1$. If all the data points fall near the line, we can regard that the data follows a normal distribution. The curve of Figure 8(b) is approximately linear because it can be considered that the fluctuation of the signal amplitude in the weak undulation conditions approximately obeys Gaussian distribution. At a significance level of 0.05 , with the Kolmogorov-Smirnov test method [7], we validate further that in the case of weak ionospheric scintillation, phase screen method simulation shows that amplitude fluctuations obey Gaussian distribution.

Figure 9 shows that in a strong scintillation condition frequency histogram of the amplitude. The curve obeys the Weibull distribution with parameters $a=b=1.5$. As it can be seen in Figure 9, the signal amplitude can be fitted quite well in the Weibull distribution. In Figure 10, we test fitting degree of signal amplitude fluctuation distribution with Weibull distribution of given parameters in the q-q figure as well with normal probability distribution in the intense fluctuation conditions. As is shown in Figure 10(a), the signal amplitude fluctuation approximately obeys the Weibull distribution. While in Figure 10(b), each amplitude point seriously deviates from the straight line. Therefore, the signal amplitude fluctuation is no longer a Gaussian distribution when it is under an intense condition. At a significance level of 0.05 , with the Kolmogorov-Smirnov test method, we validate further that in the case of strong ionospheric scintillation, phase screen method simulation shows that amplitude fluctuations obey the Weibull distribution.

To sum up, the distribution of the signal amplitude's fading is closely linked to the intensity of the fluctuation of the ionosphere. With the intensifying of the ionospheric fluctuation, the fading of the signal amplitude has undergone a transition from a Gaussian distribution to the Weibull distribution.

2.4.2. Phase Scintillation's Probability Distribution. In the case of weak scintillation, the frequency histogram of the signal phase distribution and normal distribution curve with parameters $\mu=0, \sigma^{2}=0.2$ is shown in Figure 11. From the figure, we can see that under the conditions of weak scintillation, the signal's maximum of the phase deviation is about 0.6 radians, and the signal is also in good agreement with normal distribution. We get the conclusion that in weak scintillation case, the random phase deviation of the signal obeys normal distribution with parameters $\mu=0, \sigma^{2}=0.2$. At a significance level of 0.05 , with the Kolmogorov-Smirnov test method, we validate further that in the case of weak ionospheric scintillation, phase screen method simulation shows that phase fluctuations obey Gaussian distribution. 


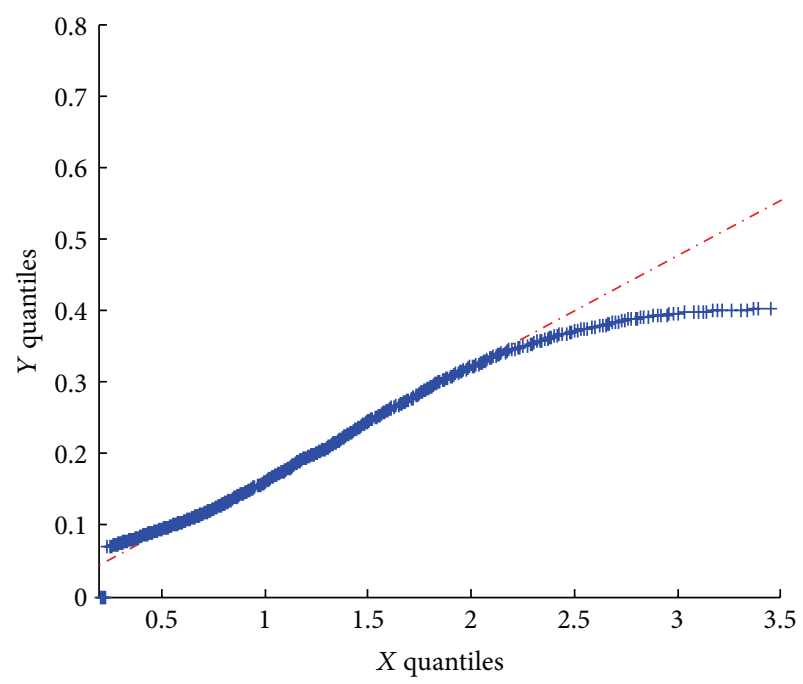

(a) Inspection of the q-q figure

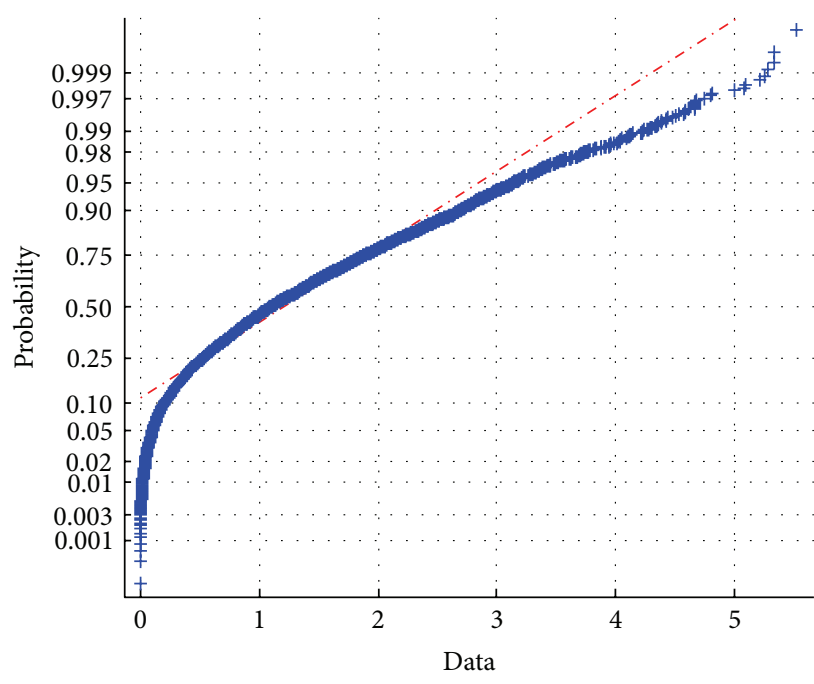

(b) Inspection in the NNP

FIGURE 10: The inspection of amplitude distribution.

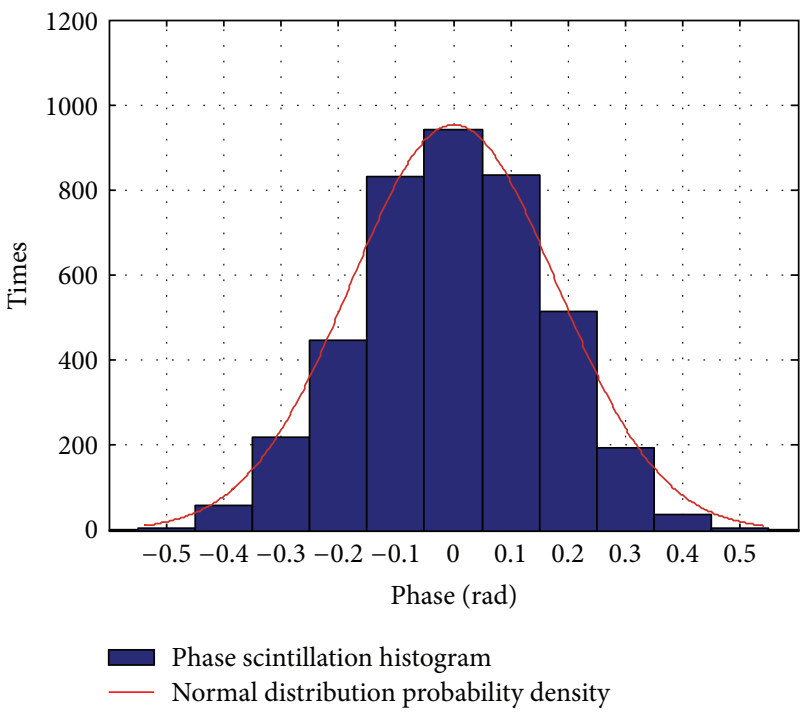

FIGURE 11: The histogram of phase scintillation and the fitting results of normal distribution.

Figure 12(a) is to verify the fitting degree of Gaussian distribution of corresponding parameters with the phase distribution of the signal using the $\mathrm{q}-\mathrm{q}$ figure. In q-q figure, the phase approximately shows linearly, and therefore, we consider that the phase scintillation belongs to the Gaussian distribution.

Figure 13 is the frequency histogram of the signal phase distribution, in the case of the strong scintillation, and the normal distribution curve with parameters $\mu=0, \sigma^{2}=0.7$. The inspection of phase scintillation is shown in Figure 14. We can see from Figure 13 that the maximum phase deviation of the signal reaches about 2 radians, but the histogram of signal phase deviation gets good fit of the normal distribution curve with the corresponding parameters $(0,0.7)$. Figure $14(a)$ is to examine fitness of the phase distribution of the signal and the corresponding parameters of the Gaussian distribution with the q-q figure. It can be seen that the curve in the q-q figure is approximately linear, and therefore, we can consider that the phase undulation obeys Gaussian distribution. Figure 14(b) is to examine fitness of the phase distribution of the signal and the Gaussian distribution with corresponding parameters with normal probability plot. Seen from the figure, the curve in the normal probability pot is approximately linear, and therefore we can consider signal phase undulation obeys Gaussian distribution in case of strong scintillation. At a significance level of 0.05 , with the Kolmogorov-Smirnov test method, we validate further that in the case of strong ionospheric scintillation, phase screen method simulation shows that phase fluctuations also obey Gaussian distribution.

The conclusion can be drawn by the phase screen method that in weak scintillation case, the signal amplitude and phase scintillation are relatively small, while its amplitude and phase fit the Gaussian distribution. In the case of strong scintillation, the Weibull distribution is a much better choice to characterize the scintillation of the signal amplitude. Phase scintillation still approximates the Gaussian distribution, while the deviation has increased than that of the weak case.

2.4.3. The Comparison with Measured Data. The amplitude and phase distribution characteristics can be calculated by statistics analysis based on the measured data of ionospheric Es layer clutter echo, which can be used as the basis to test whether the simulation results of the phase screen method can reflect real ionospheric characteristics.

The frequency distribution histogram of an amplitude of 6 batches of real ionospheric echo is shown in Figure 15. The abscissa is the normalized amplitude of the ionospheric echo, and the ordinate is the occurrence frequency of the corresponding amplitude. Red curve is a Weibull distributions with certain parameters, from which we can see 


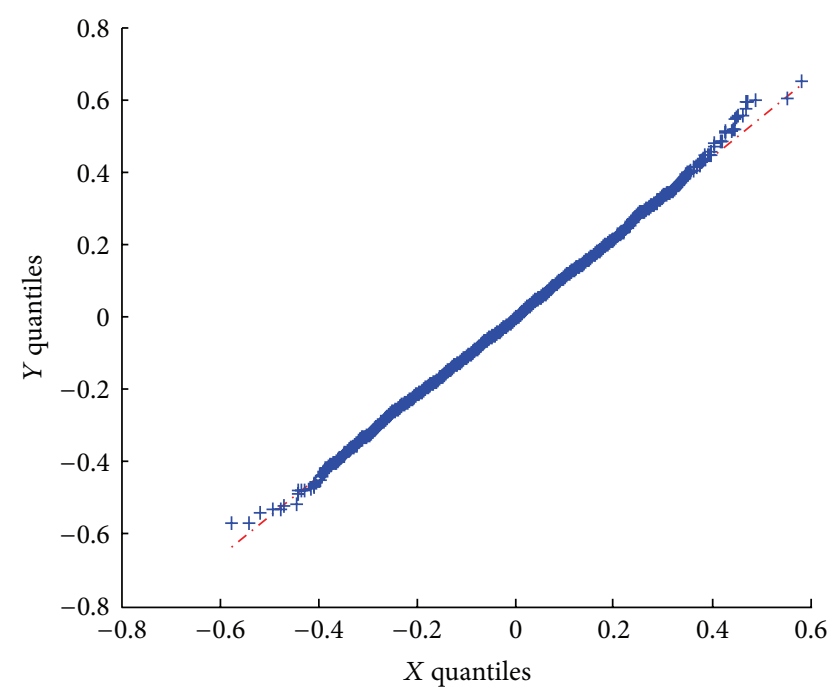

(a) Inspection of the q-q figure

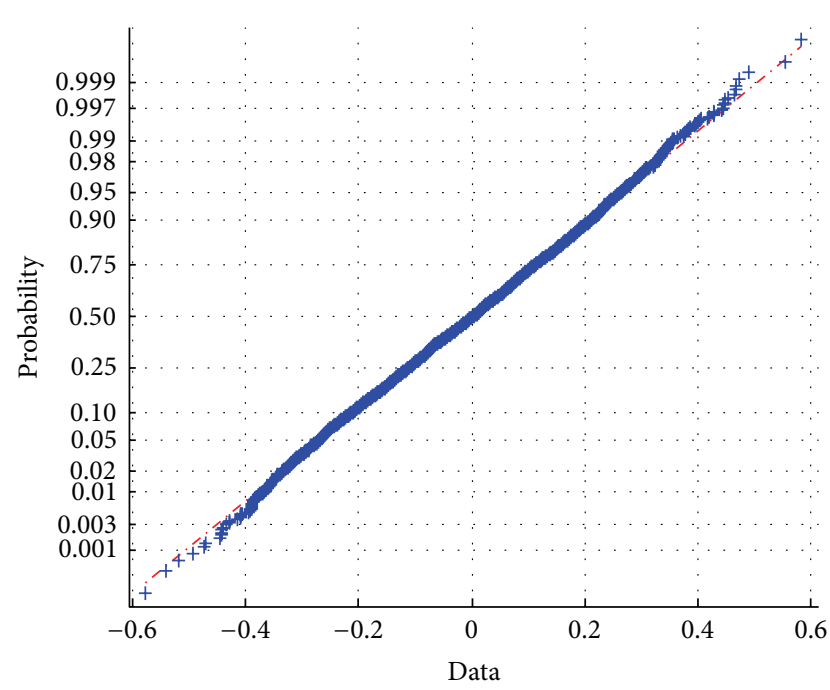

(b) Inspection in the NNP

FIGURE 12: The inspection of phase distribution.

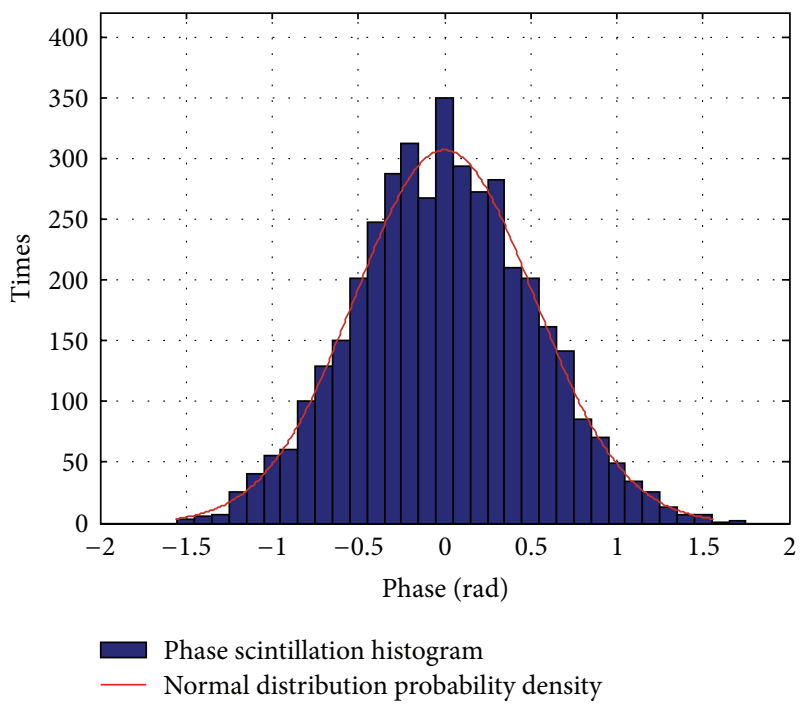

FIGURE 13: The histogram of phase scintillation and the fitting results of normal distribution.

that the amplitude scintillation approximately obeys the Weibull distribution and that amplitude distribution of the signal derived from our simulation in the strong scintillation basically coincides with the previous one. At a significance level of 0.05 , we further verify the validity of the conclusion that the amplitude fluctuations of measured data obey the Weibull distribution by using the Kolmogorov-Smirnov test method.

We simulate ionospheric echo by phase screen method, obtain the phase of the simulated echo signal, and then process statistical analysis to finally get the phase distribution of the ionosphere echo signal. Then, we do the same statistical analysis to the corresponding measured ionospheric echo signal to obtain its phase fluctuation frequency distribution histogram. The phase distribution histogram of the simulated echo signal is shown in Figure 16. We can get the phase distribution of the actual ionospheric echo after we analyze the measured data, which is shown in Figure 17.

As can be seen from Figure 16, due to the ionosphere, the phase of the echo signal approximately subjects to uniform distribution. Figure 17 shows the histogram statistical analysis of a phase of 6 batches of measured data. As can be seen from Figure 17, ionospheric echo signal's phase also approximately obeys uniform distribution, which is consistent with the conclusions of theoretical simulation. In order to confirm that the phase screen simulation results and the actual data come from the same distribution, we test six sets of data available with the q-q figure. Figure 18 shows the results of a set of data with the q-q figure.

As can be seen from Figure 18, ionospheric echoes' phase distribution derived from phase screen method is able to fit the actual ionospheric echo data, that is, the models created by the phase screen method can preferably simulate the echoes' phase. At a significance level of 0.05, we further verify the validity of the conclusion that the echo's phases of measured data obey uniform distribution by using the Kolmogorov-Smirnov test method. To sum up, in strong scintillation condition, the scintillation of amplitude and phase by simulation is close to the statistical results of actual data and basically characterize the impact ionospheric irregularities on high-frequency radio waves.

\section{Ionospheric Es Layer Clutter Model Based on Multiphase Screen Method}

3.1. The Proposition of Ionospheric Es Layer Clutter Model. Es layer is an ionization cloud with a variety of different shapes that appear in the height of the E layer, and its electronic peak concentrations are often many times higher than the concentration of the E layer around. Es layer appears in 


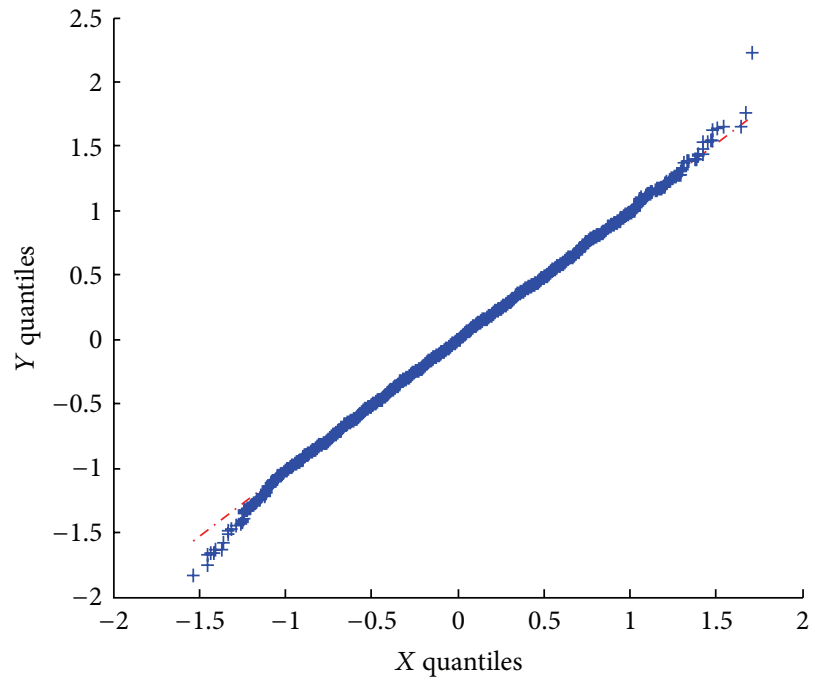

(a) Inspection of the q-q figure

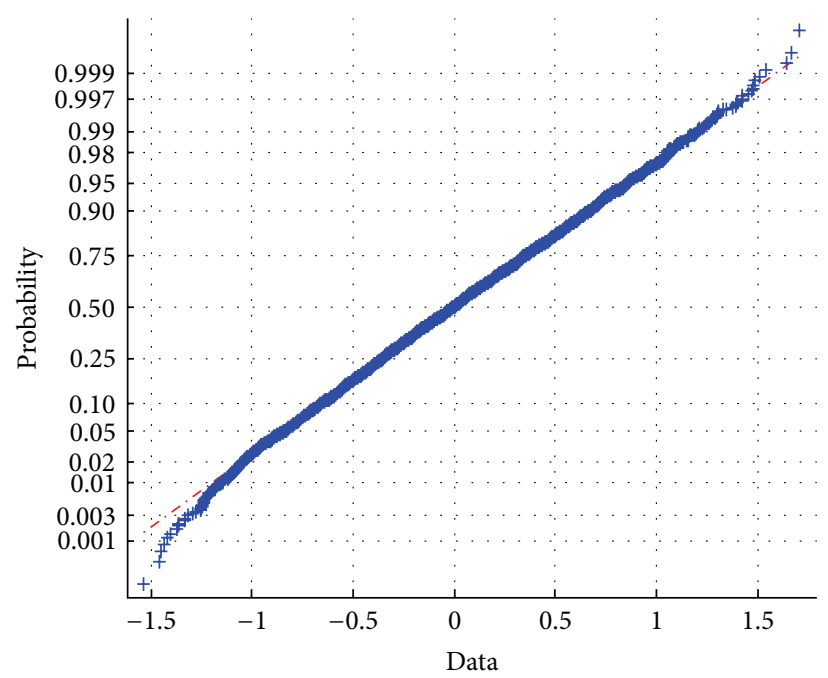

(b) Inspection in the NNP

FIGURE 14: The inspection of phase distribution.

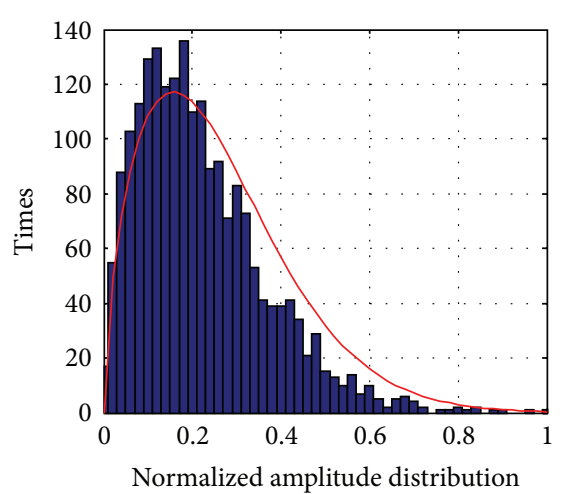

Data 1

- Weibull $(a=1.512, b=1.603)$

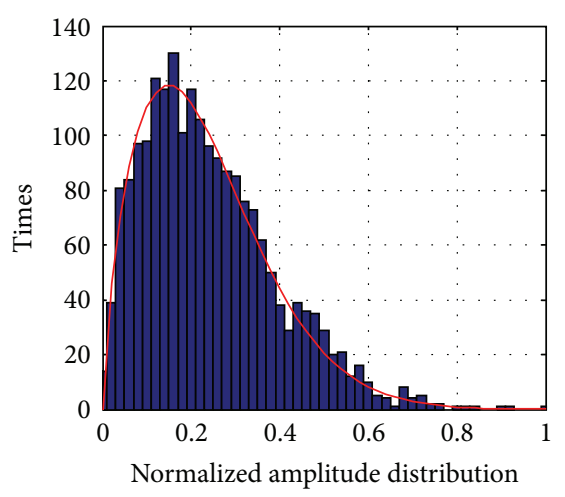

Data 4

Weibull $(a=1.368, b=1.612)$
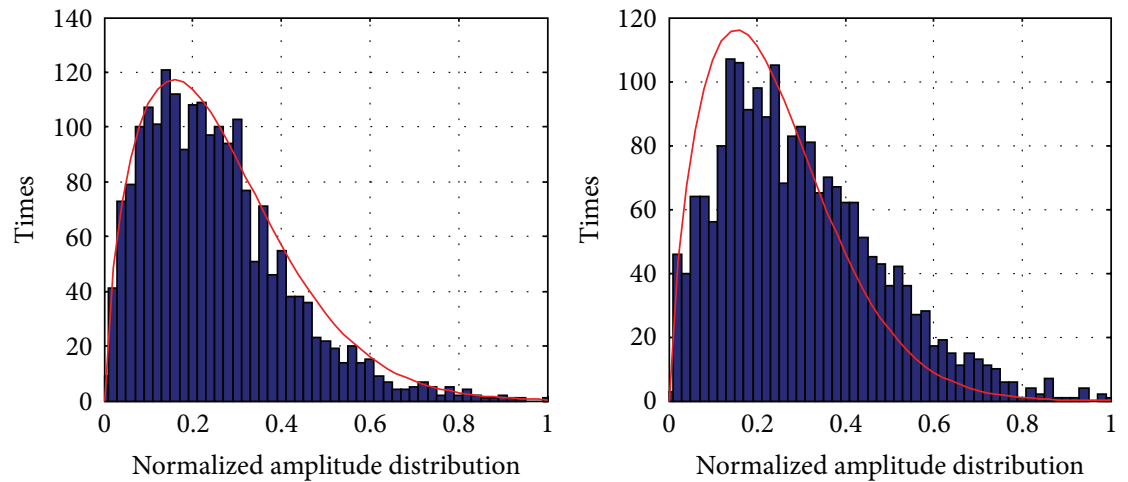

Data 2

Weibull $(a=1.648, b=1.592)$

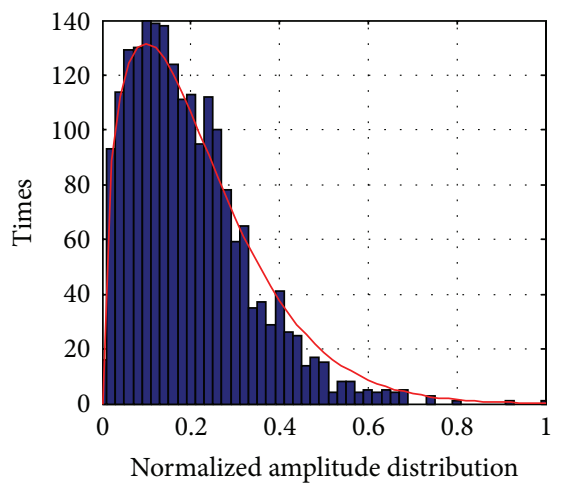

Data 5

Weibull $(a=1.734, b=1.425)$

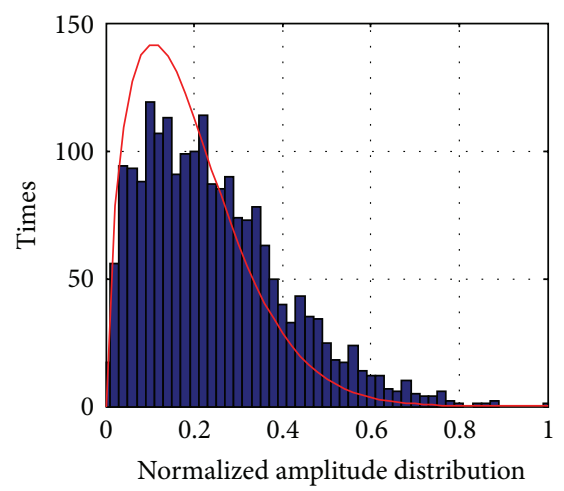

Data 6

Weibull $(a=1.691, b=1.526)$

FIGURE 15: Actual measured ionospheric echo amplitude histogram. 


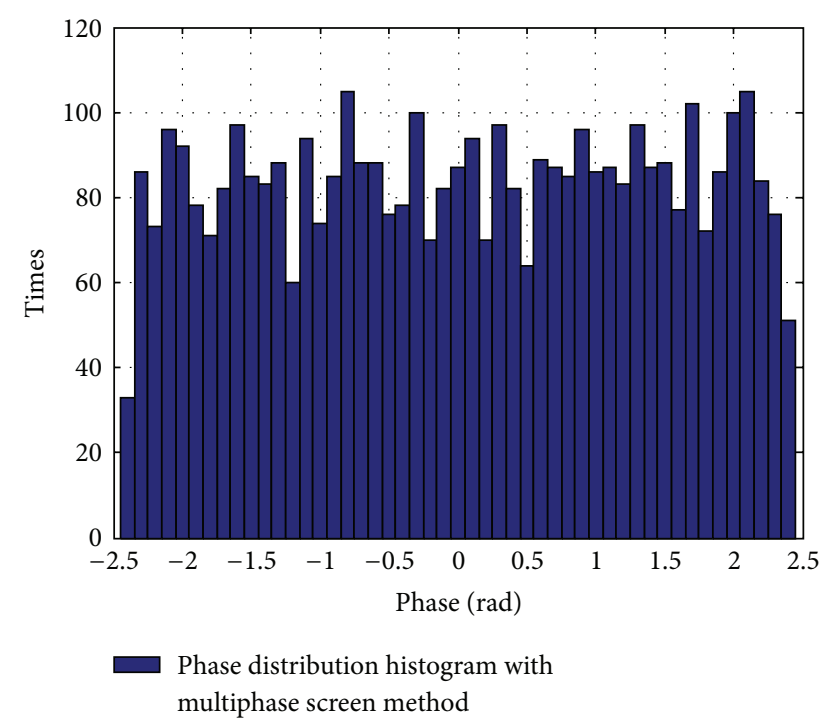

FIgURE 16: The phase distribution of ionospheric echo by phase screen method simulation.

the corresponding state of strong scintillation of ionosphere. According to the previous study of the propagation characteristics in the ionospheric Es layer, under strong scintillation conditions, we can conclude the signal amplitude scintillation probability distribution of radio signals that through the ionosphere can use the Weibull distribution to overfit, and phase scintillation probability distribution approximates to a Gaussian distribution. Using the results obtained by the multiphase screen method, taking into account of the variation in the height of the ionospheric reflection wave reflection points, we give a new reflected wave model of the ionosphere Es layer.

For HF surface wave radar, the difference frequency signal of uniform motion target's echo after passing mixer is

$$
s(t)=A \cdot \sum_{n=0}^{N-1} e^{j \cdot 2 \tau \cdot\left(f_{0} \tau-\left(a \tau^{2} / 2\right)+a \tau \tau\left(t-n T_{s}\right)\right)},
$$

where $A$ represents echo amplitude, $\tau=2\left(R_{0}+v t\right) / c$ represents the echo delay, $R_{0}$ is the initial distance of the target distance to radar, $v$ shows radial velocity of the target relative to the radar, $a$ is the chirp rate, and $T_{s}$ is the sweep cycle.

In contrast, the ionospheric Es layer clutter echo is more complex. Three main aspects need to be considered: there is a random amplitude fluctuation in clutter echoes' difference frequency signal of ionospheric Es layer; there is a random phase fluctuation in clutter echoes' difference frequency signal of ionospheric Es layer; the echoes' height of reflection point in ionospheric Es layer clutter may randomly change. When waves vertically incident to the ionosphere, refractive index in each height under the reflecting surface will generate a Doppler frequency shift as time changes, and the movement of the reflecting surface will also produce Doppler shift.

According to previous theoretical analysis, simulation data analysis, and the conclusion of the actual data analysis, we establish a new echo model as follows.
Step 1. In the difference frequency signal of the standard uniform motion target, we superimpose on a random fluctuation amplitude. The distribution of the random fluctuations obeys the Weibull distribution.

Step 2. In the difference frequency signal of the standard uniform motion target, we superimpose on a random fluctuation phase. The distribution of the random fluctuations obeys Gaussian distribution.

Step 3. The height of ionospheric echo reflection point is a random variable. We can handle this change by dividing the whole coherent cycle sweep cycle $M$ into $N$ portions. In each section, the target velocity corresponding to a single sweep cycle of ionospheric echoes (i.e., the height of the ionospheric reflection point) is fixed, and the target moving speed is different in the different sweep cycle of ionospheric echoes. All of the $M / N$ targets' speed corresponding to theionospheric echoes within the $M / N$ sweep cycles obey Gaussian random distribution which takes a fixed speed as the mean value. The corresponding $N$ fixed speed uniformly distributes in $(-v, v)$, typically $v=10 \mathrm{~m} / \mathrm{s}$.

According to the ionospheric echo model established previously, the difference frequency signal of the Es layer ionospheric clutter echoes after mixing is given by the following formula:

$$
\begin{aligned}
s(t)= & \text { Amplitude }(t) \cdot A \\
& \cdot \sum_{n=0}^{N-1} e^{j \cdot\left(2 \pi \cdot\left(f_{0} \tau-\left(a \tau^{2} / 2\right)+a \tau\left(t-n T_{s}\right)\right)+\text { Phase }(t)\right)},
\end{aligned}
$$

where Amplitude $(t)$ represents the amplitude of the Weibull random fluctuations, Phase $(t)$ represents the Gaussian random fluctuations in the phase, $v$ presents ionospheric echoes variation in the height of the reflection point, and $R_{0}$ corresponds to Es layer height in delay characterization $\tau=$ $2\left(R_{0}+v t\right) / c$.

\subsection{The Comparison of Simulating Ionospheric Es Layer} Clutter and the Actual Ionospheric Es Layer Clutter. The range of ionospheric Es layer clutter of high-frequency ground wave radar is generally from $100 \mathrm{~km}$ to $200 \mathrm{~km}$, and to reduce sea clutter's impact on the accuracy of statistical characteristics analysis of the ionosphere clutter, we use a method based on eigenvalue decomposition to suppress the sea clutter composition in the time domain signal [8] and then do the statistical analysis of remaining ionospheric Es layer clutter. According to the model of ionosphere Es layer built in the last section, the simulation can produce the ionospheres' Es layer clutter after selecting the height value corresponds to the Es layer. At this time, the stochastic amplitudes obey the Weibull distribution with the parameters of $(7,2.8)$, and the phase of stochastic volatility obey Gaussian distribution with the parameters of $(0,0.4)$, wherein the unit of phase distribution's parameter 0.4 is radians. Figure 19(a) shows the spectrum of simulating ionospheric Es layer clutter corresponding to the range gate at the reflection height of Es layer. For 

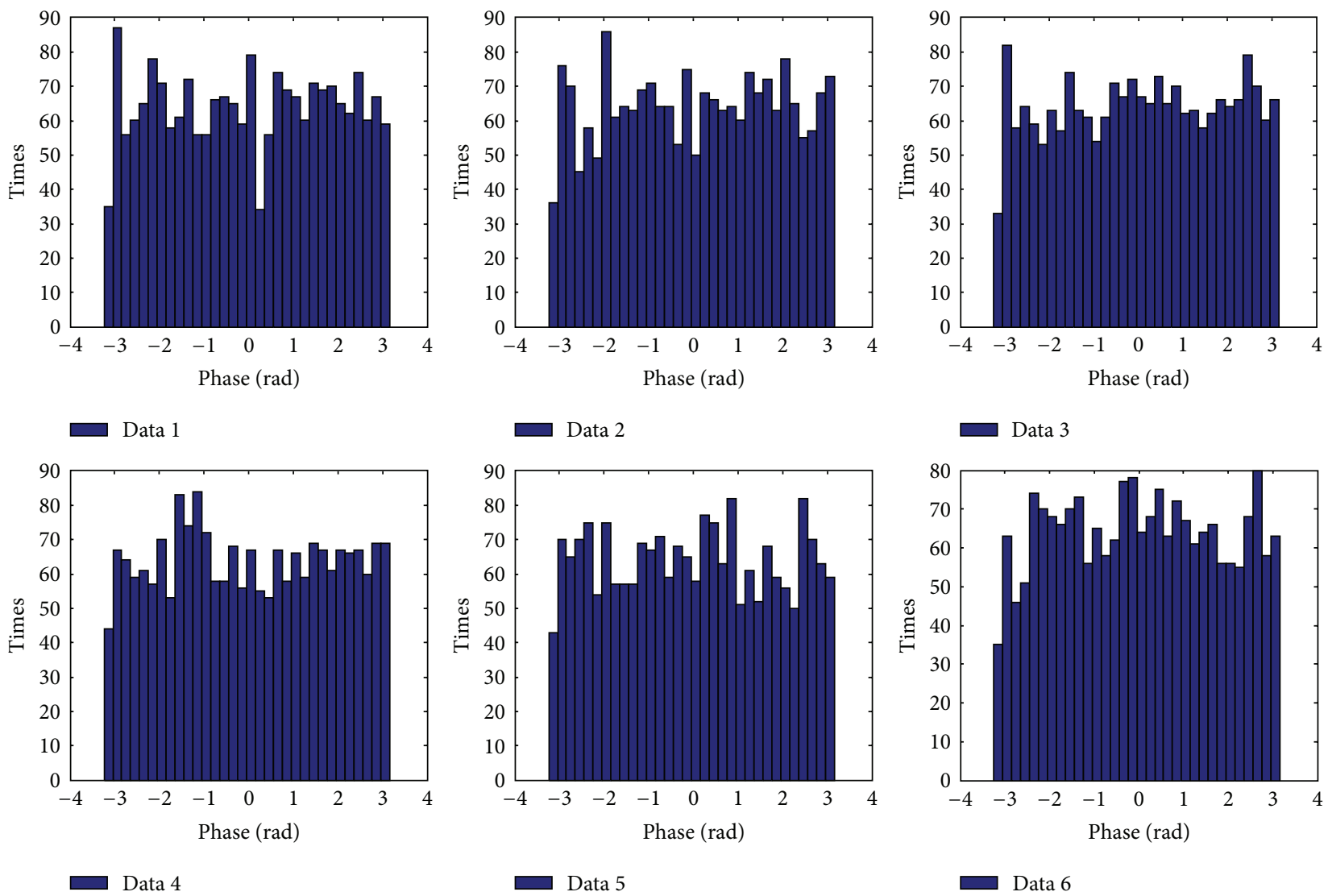

FIGURE 17: The phase distribution of the actual ionospheric echo.

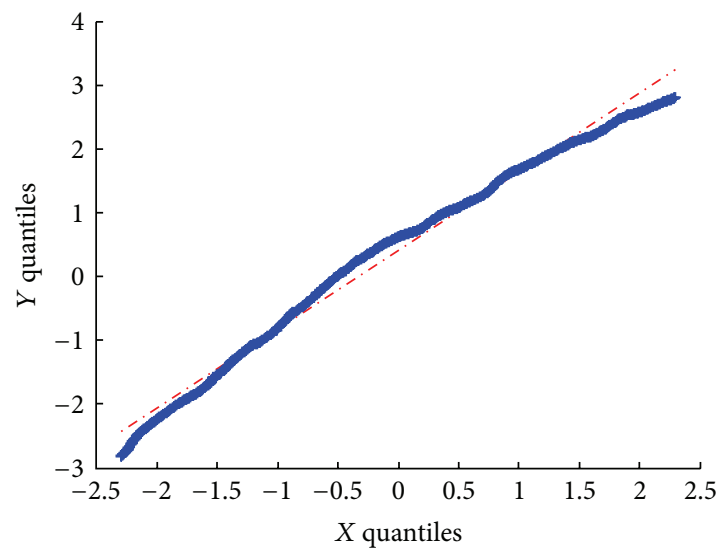

FIGURE 18: The examination of the echo phase distribution.

the comparison, Figure 19(b) shows the spectrum of actual ionospheric Es layer clutter corresponding to the range gate at the height of Es layer.

From the comparison of Figures 19(a) and 19(b), we can intuitively see that the spectrums of simulating and actual Es layer ionospheric clutter are very similar. The centered section of the spectrum of the simulating Es layer ionospheric clutter is obviously raised, which is in accordance with the actual one. From Figure 19(a), the centered raised section covers the range of $-0.25 \mathrm{~Hz} \sim 0.25 \mathrm{~Hz}$, which is exactly the range corresponding to the ship targets detected by HF surface wave radar. The amplitudes of the centered raised section are nearly $30 \mathrm{~dB}$ higher than the amplitudes of neighbouring sections which do not include Es layer clutter. From Figure 19(b), we can see the same phenomenon; the target and Bragg crest are both submerged by the ionospheric Es layer clutter.

From the comparison and analysis of simulating and actual clutter echoes, we can see that the proposed model can represent the actual Es layer ionospheric clutter well.

\section{The Ionospheric Es Layer Clutter Suppression Based the JDL Algorithm}

Space-time adaptive processing (STAP) is a kind of low complexity and effective way for using training samples to suppress clutter, having become an important direction of research scholars from various countries and has been used in airborne radar [9-15]. Similar to ground clutter, there are certain orientation-Doppler frequency characteristics in high-frequency surface wave radar ionospheric clutter; so, using STAP to suppress ionospheric clutter becomes possible. The traditional fully space-time adaptive algorithm has a large number of degrees of freedom. It is impossible for high-frequency surface wave radar to have enough secondary data samples to estimate the covariance matrix, and 


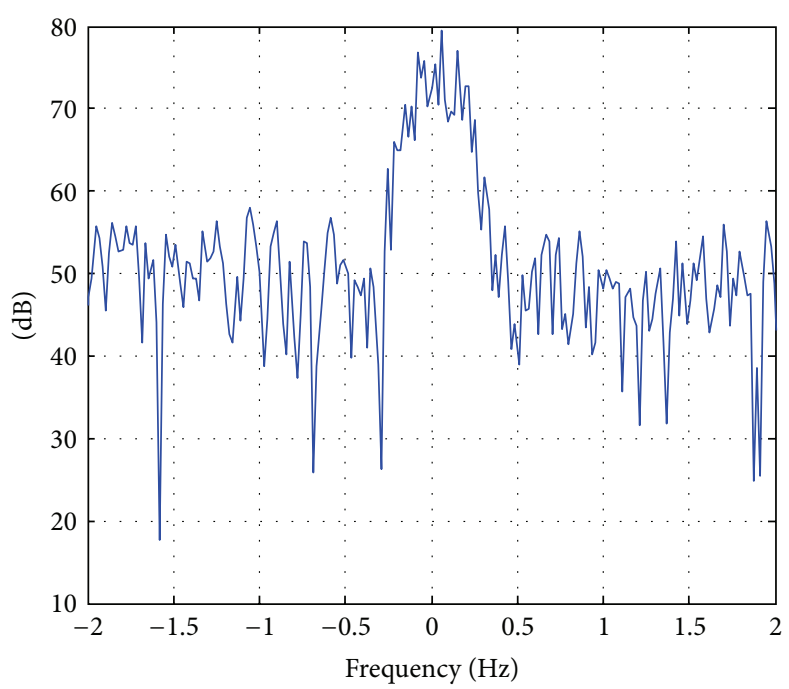

(a) Clutter spectrum of simulating

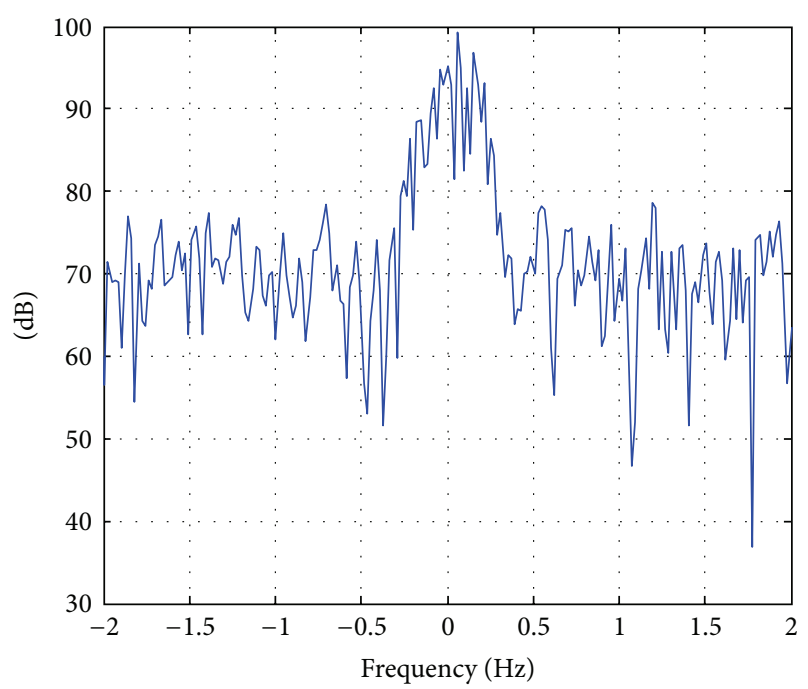

(b) Clutter spectrum of actual

FIGURE 19: Spectrums of simulating and actual Es layer clutter.

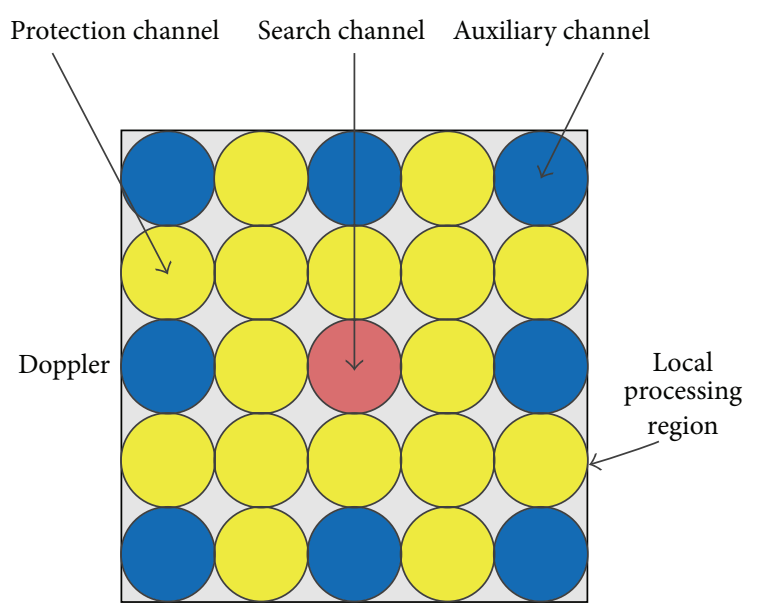

FIgURE 20: JDL schematic diagram.

the computation load is too large. Therefore, in this section we inject an ideal point target to the simulated ionospheric clutter data and measured ionospheric clutter data, majoring in a kind of dimension reduced STAP algorithm and JDL's ability to suppress the ionospheric clutter and find the target.

4.1. Joint Domain Localized (JDL) Algorithm Principle. Spacetime adaptive processing is using the training samples close to the range bin to be detected to estimate clutter and noise covariance matrix $R$, and according to linearly constrained minimum variance criteria (LCMV) to estimate adaptive weights $w$, and then to weigh the received data to maximize SNR. Covariance matrix is given by

$$
R=\frac{1}{K} \sum_{i=1}^{K} X_{i} \cdot X_{i}^{H},
$$

where $X_{i}$ is a training sample data.
Weight vector can be given by

$$
w=\mu R^{-1} v=\mu\left(R^{-1 / 2}\right)\left(R^{-1 / 2} v\right),
$$

where $\mu$ is a complex normalized constant.

The JDL algorithm's mainly take advantage of the transformation vector $T$ to transform the space-time data to the angle-Doppler domain and select a small local area for adaptive processing. It is a kind of dimension reduced algorithm of the STAP processing, solving the lack of training samples and excessive computation load.

$S_{s}$ and $S_{t}$, respectively, are space steering vector and time steering vector. Elements of theirs are the discrete Fourier transform coefficients. Therefore, the inner product of the spatial and temporal steering vector is equivalent to 2-D DFT, and then, the process of transforming the received data from space-time domain to the $i$ th angle bin $w_{s i}$, the $j$ th Doppler bin, we concern can be expressed as

$$
\widetilde{X}=\left(S_{s}\left(w_{s i}\right)\right) \otimes\left(S_{t}\left(w_{t j}\right)\right)^{H} X
$$

The JDL algorithm transforming matrix can be represented as

$$
T=\left(\begin{array}{c}
{\left[S_{s}\left(w_{s i}\right) \otimes S_{t}\left(w_{t j}\right)\right]^{T}} \\
{\left[S_{s}\left(w_{s i}\right) \otimes S_{t}\left(w_{t j}+w_{k}\right)\right]^{T}} \\
\vdots \\
{\left[S_{s}\left(w_{s i}\right) \otimes S_{t}\left(w_{t j}+(q-1) w_{k}\right)\right]^{T}} \\
\vdots \\
{\left[S_{s}\left(w_{s i}+(p-1) w_{n}\right) \otimes S_{t}\left(w_{t j}+(q-1) w_{k}\right)\right]^{T}}
\end{array}\right)^{T}
$$

where $w_{n}$ and $w_{k}$, respectively, represent angle and Doppler interval, $p$ represents the number of adjacent angle bins, and 


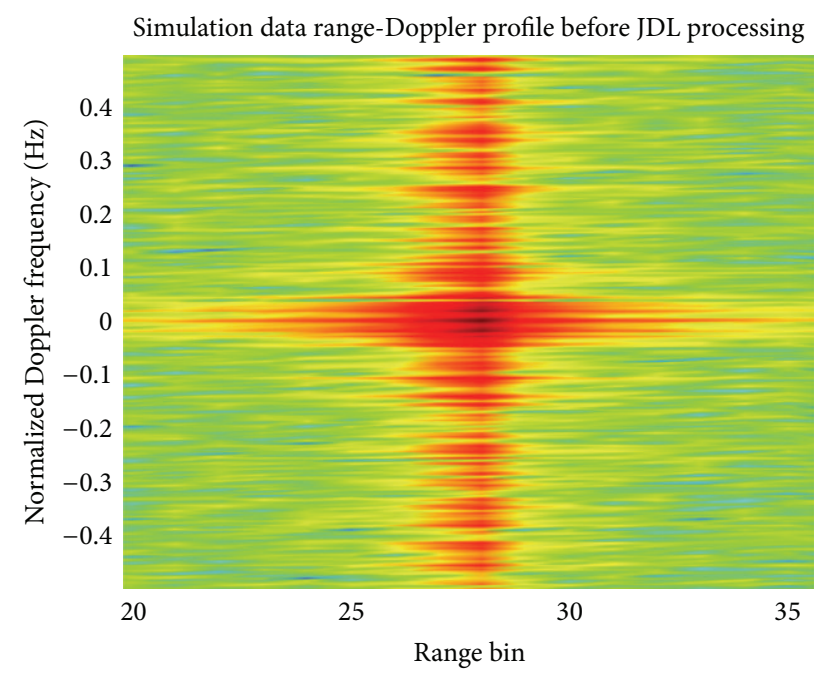

(a) Range-Doppler profile before the JDL algorithm

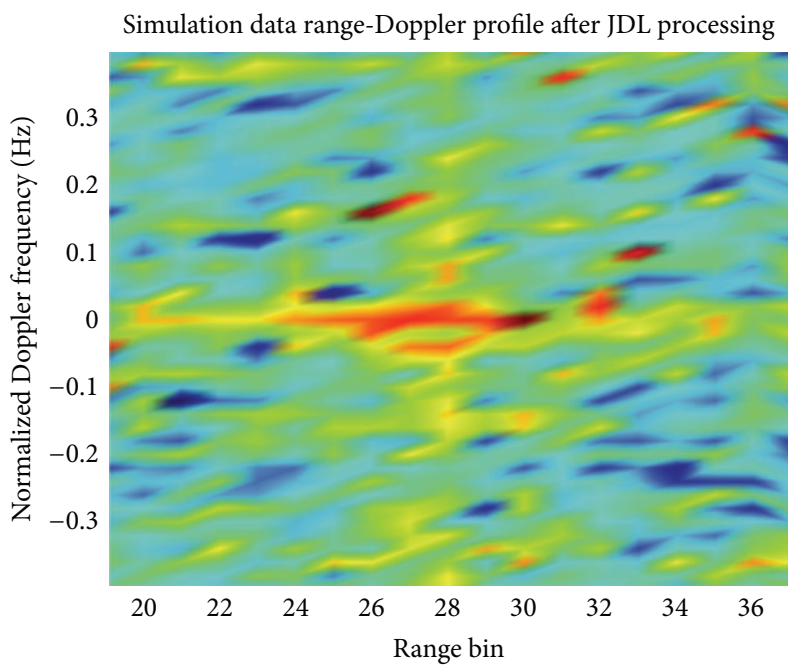

(b) Range-Doppler profile after the JDL algorithm

FIGURE 21: Angle-Doppler profile before and after the JDL algorithm.

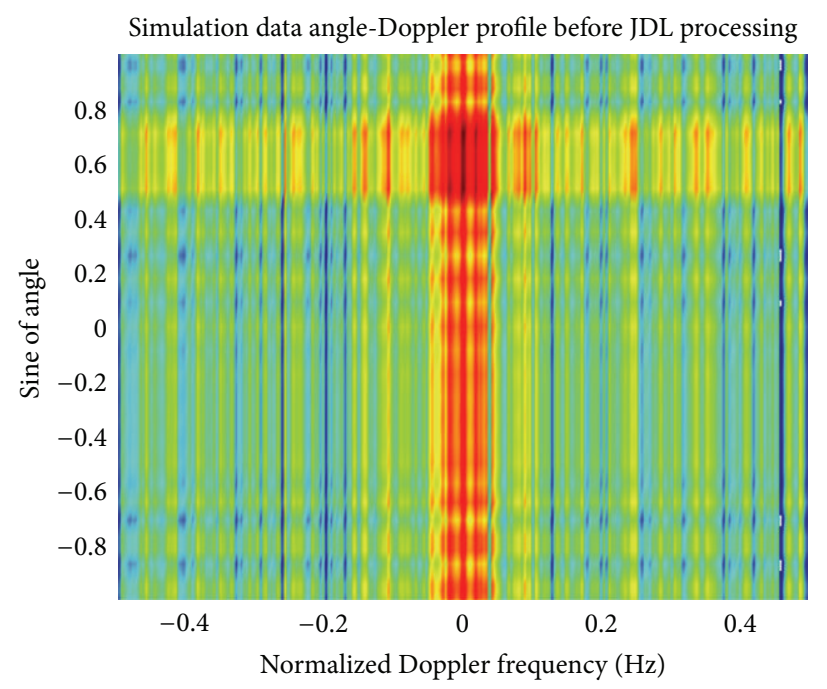

(a) Angle-Doppler profile before the JDL algorithm

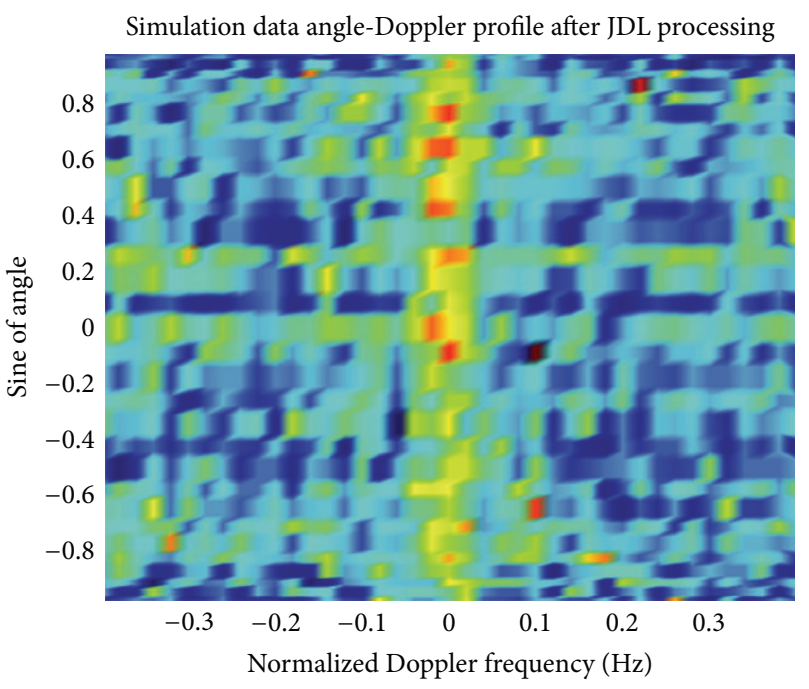

(b) Angle-Doppler profile after the JDL algorithm

FIGURE 22: Angle-Doppler profile before and after the JDL algorithm.

$q$ represents the number of adjacent Doppler bins. Schematic diagram 20 is as follows.

In Figure 20, the red channel is the search channel, the blue channel is the auxiliary channel, and the yellow channel is the protection channel. The selection of a protection channel in angle-Doppler domain may be effective in preventing the spread of the target signal to clutter covariance matrix, affecting the actual detection performance.

When selecting the $3 \times 3$ local shown in Figure 20, space -time transformation matrix can be expressed as

$$
\begin{aligned}
T= & {\left[S_{t}\left(w_{t, j-2}\right) ; S_{t}\left(w_{t, j}\right) ; S_{t}\left(w_{t, j+2}\right)\right] } \\
& \otimes\left[S_{s}\left(w_{s, i-2}\right) ; S_{s}\left(w_{s, i}\right) ; S_{s}\left(w_{s, i+2}\right)\right] .
\end{aligned}
$$

The transformed space-time steering vector is

$$
\widetilde{v}=T^{H} \cdot v .
$$

The receiving data vector is

$$
\widetilde{X}=T^{H} \cdot X .
$$

The corresponding adaptive weight vector is

$$
\widetilde{w}=\mu \widetilde{R}^{-1} \widetilde{v} .
$$

4.2. The JDL Algorithm Performance Analysis Based on Es Layer Cutter Simulation Model. Parameter setting of the simulation data: the carrier frequency $f_{c}=4.05 \mathrm{MHz}$, the sampling frequency $f_{s}=10 \mathrm{kHz}$, the bandwidth $B=20 \mathrm{kHz}$, 


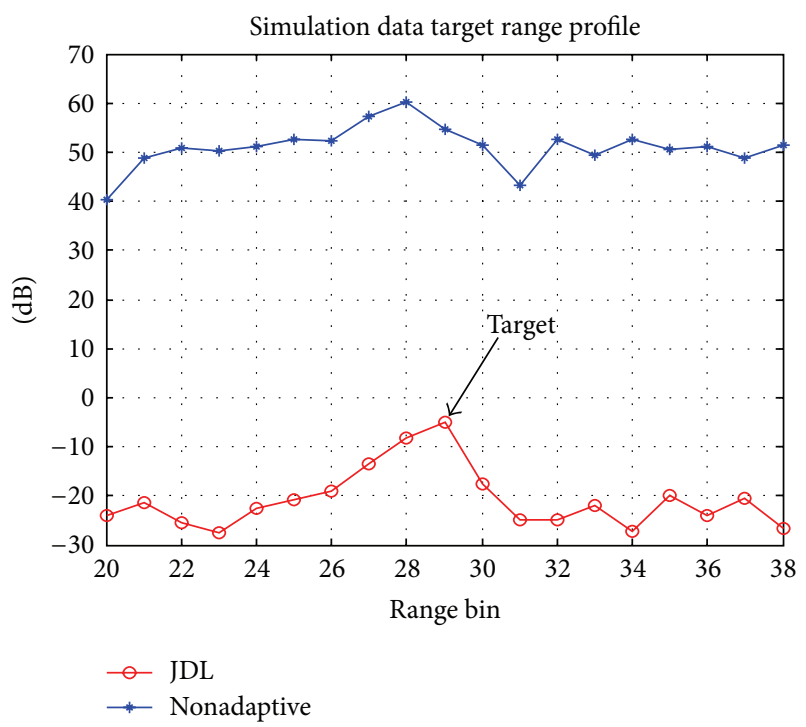

(a) Target range profile before and after the JDL algorithm

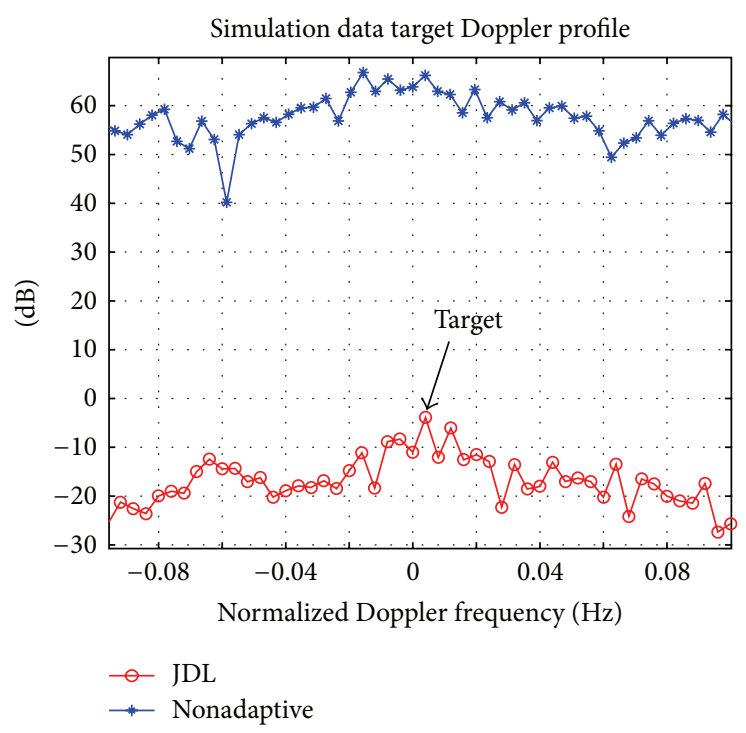

(b) Target Doppler profile before and after the JDL algorithm

FIGURE 23: Target range profile and Doppler profile before and after the JDL algorithm after injecting a target.

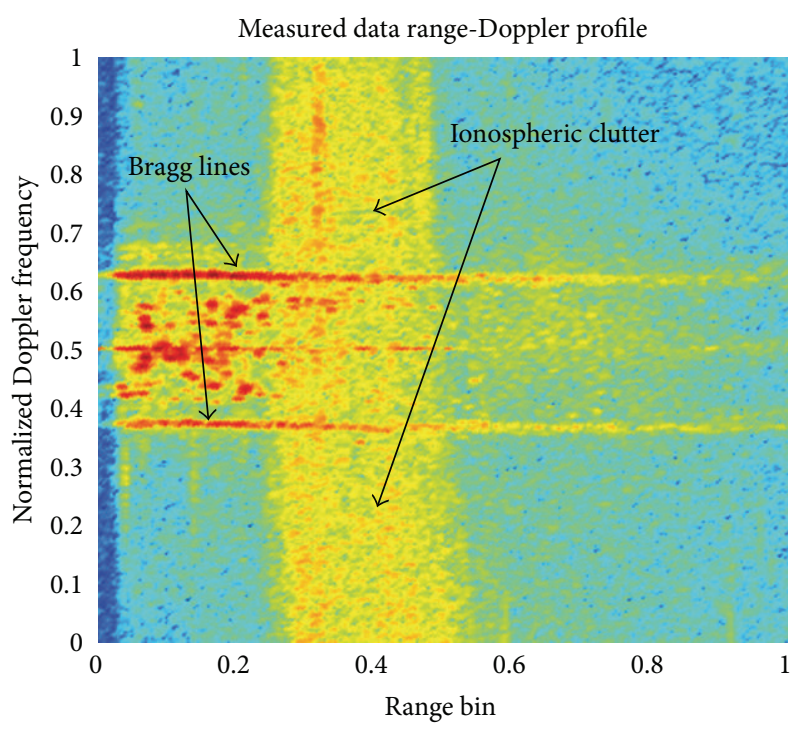

(a) The distance-Doppler profile without JDL algorithm

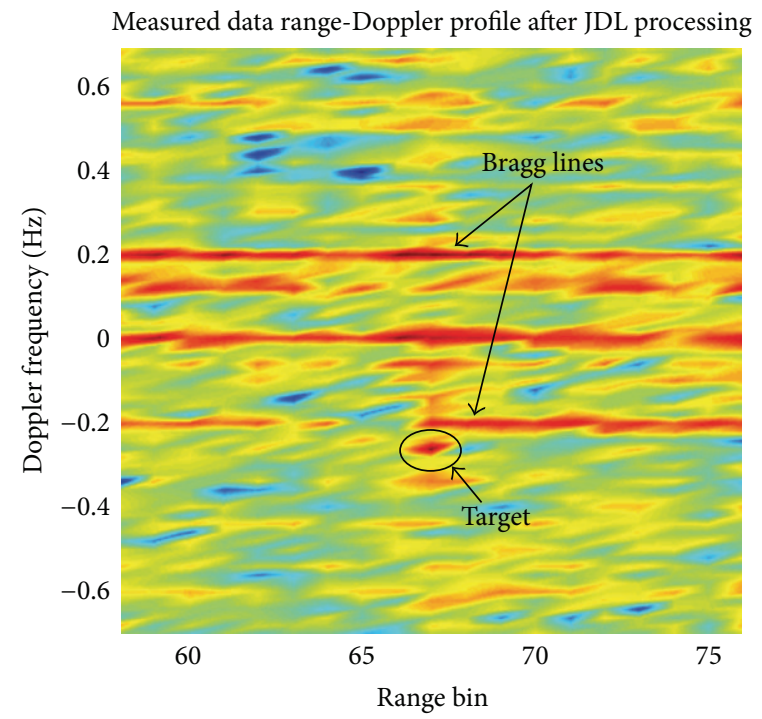

(b) The range-Doppler profile after JDL algorithm

FIGURE 24: Range-Doppler profile before and after JDL.

the pulse period is $20 \mathrm{~ms}$, the number of array elements $N=$ 32, the accumulated pulse numbers $M=4096$, the angle between the target incident direction and the array normal is $30^{\circ}$, the target normalized Doppler frequency $f_{\text {-Doppler }}=$ $0.004 \mathrm{~Hz}$, target range bin is 29, Es layer height $h=200 \mathrm{~km}$, and signal to clutter ratio (SCR) is $-10 \mathrm{~dB}$. Based on the established ionospheric Es layer clutter model, when the target is not injected, comparing the range-Doppler profile before and after the JDL algorithm processing, as Figures 21(a) and 21(b) show.
Figures 22(a) and 22(b) compared the angle-Doppler output before and after the JDL algorithm processing when the target is not injected.

From Figures 21 and 22, we can see that the simulated ionospheric clutter exists obvious broadening in range, angle and Doppler profile after conventional processing, while after JDL processing, the clutter is significantly reduced. So, JDL algorithm can achieve good suppression performance for simulated ionospheric clutter. Figures 23(a) and 23(b) show the target range profile and the target Doppler profile before 


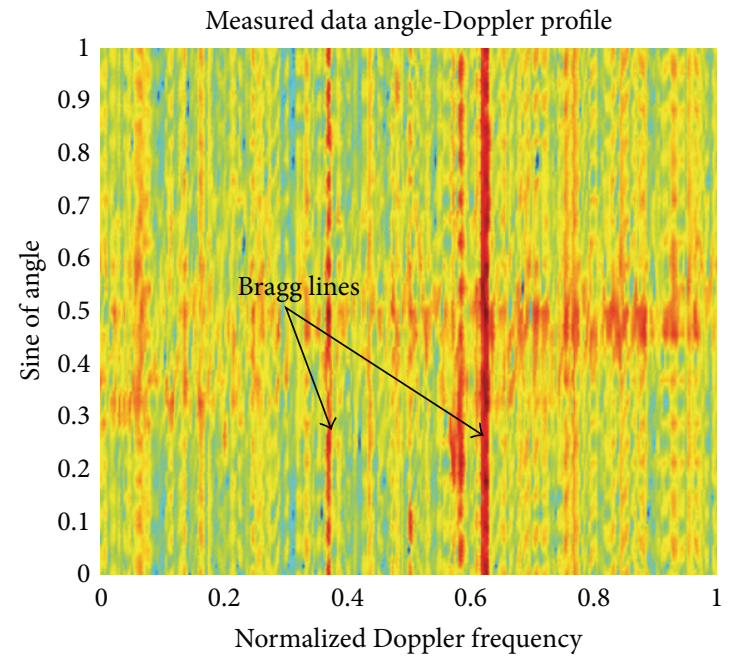

(a) Angle-Doppler profile before JDL algorithm

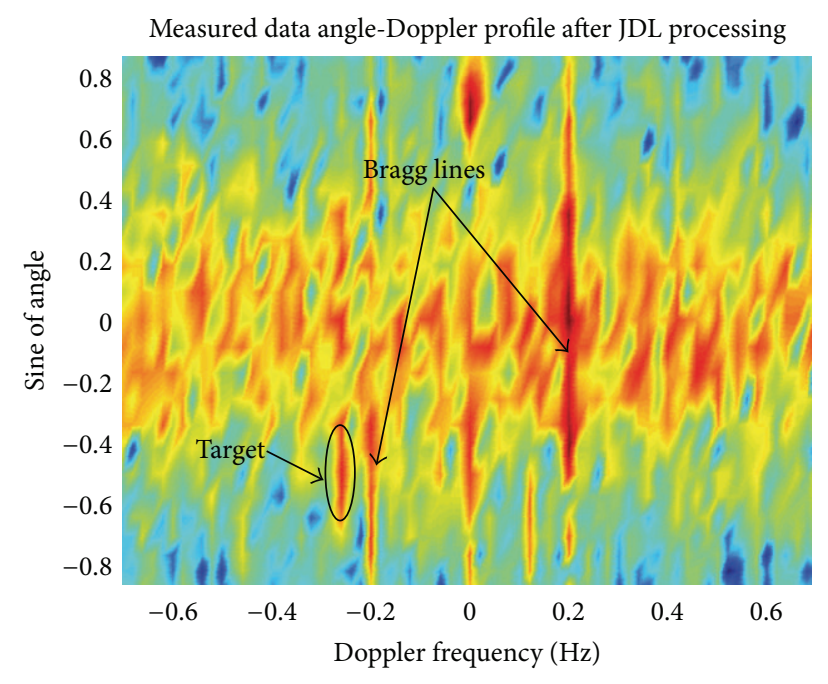

(b) Angle-Doppler profile after JDL algorithm

FIGURE 25: Angle-Doppler profile before and after JDL algorithm.

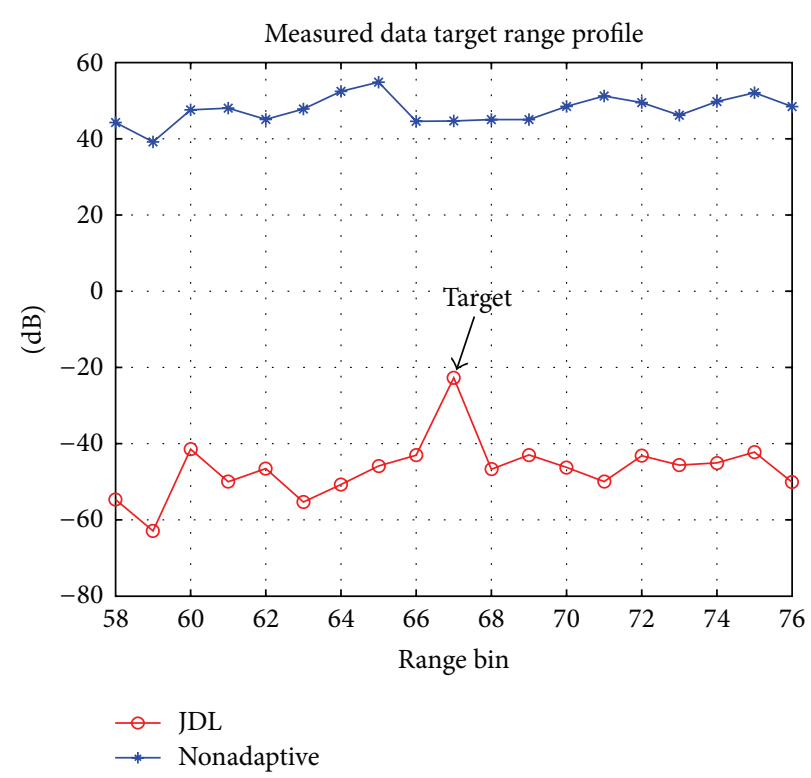

(a) Target range profile before and after JDL algorithm

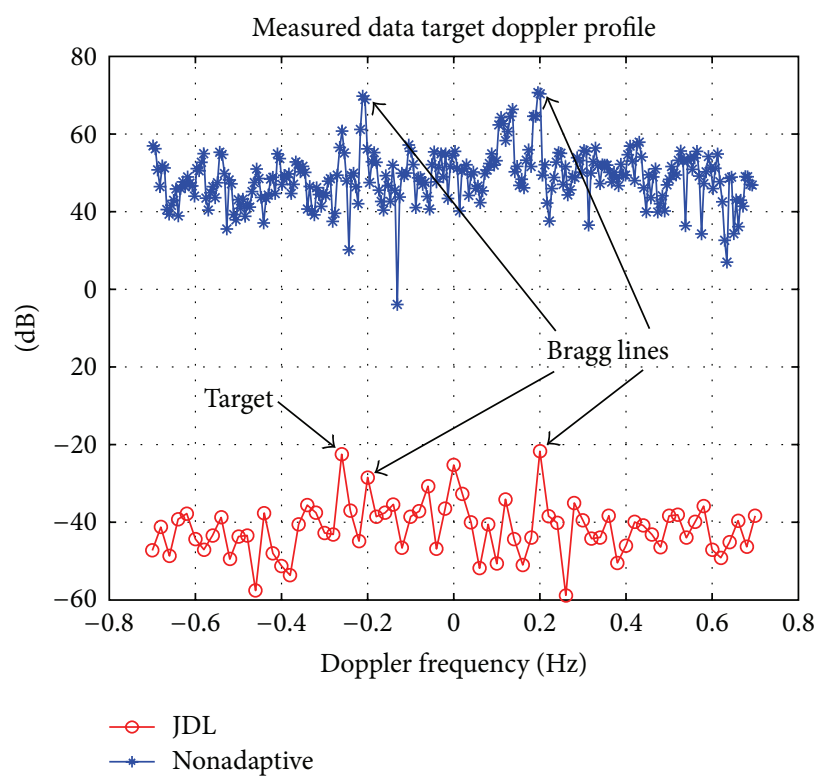

(b) Target doppler profile before and after JDL algorithm

FIGURE 26: Target range profile and doppler profile before and after the JDL algorithm after injecting a target.

and after the JDL algorithm processing after injecting an ideal target for simulated ionospheric clutter.

We can see from the simulation results in Figure 23 that SCR is $-10 \mathrm{~dB}$ before JDL processing, and SCR in the range and Doppler dimension is greatly improved after JDL processing. This further illustrates that the JDL algorithm can effectively suppress the ionosphere clutter and detect target submerged by ionospheric clutter.

4.3. Analysis of the JDL Algorithm Performance Based on Measured Data. Parameter settings for the measured data: carrier frequency $f_{c}=4.05 \mathrm{MHz}$, coherent integration time $T=184.32 \mathrm{~s}$, the angle between the target incident direction and the array normal is $-30^{\circ}$, target normalized Doppler frequency $f_{\text {-Doppler }}=-0.26 \mathrm{~Hz}$, target range bin is 69 , and SCR $=-10 \mathrm{~dB}$. Figure 24(a) shows the rangeDoppler profile of a batch of HF ground wave radar data in actual measurement after regular processing. Figure 24(b) is the range-Doppler profile after JDL processing, and the range bin of the injected target is 69 .

Figure 25(a) shows the angle-Doppler profile of measured data after regular processing; Figure $25(\mathrm{~b})$ is the angleDoppler profile after JDL processing. Figures 26(a) and 26(b) show the target range profile and the target Doppler profile 
before and after the JDL algorithm processing after injecting an ideal target for measured ionospheric clutter.

We can see, from the JDL processing results of the previously measured data, that the SCR in range and Doppler dimension are greatly improved, further illustrating the effectiveness of the JDL algorithm in suppressing the ionosphere clutter and verifying the reasonableness of the built clutter model.

\section{Conclusions}

An ionospheric Es layer clutter model based on multiphase screen method with DFT fast implementation was proposed, and then, the Es layer clutter was suppressed by STAP method in this paper. First, the basic principle of the multiphase screen method in the disturbed layered ionosphere was analyzed, and the DFT-based fast algorithm was proposed. Then, we utilized the multiphase screen method to obtain statistical analysis results as well as took into account the height of the reflection points' variation in the ionospheric Es layer to create a new reflecting model in the ionospheric Es layer. We also compared it with the measured data and analysis to verify the reasonableness and correctness of the established clutter model. Finally, based on the ionospheric clutter model, the newly developed descending dimensional JDL algorithm was used to suppress the Es layer clutter and simulated with measured data to provide the validity of this algorithm to suppress the ionospheric clutter. The simulation results showed that the established model can show the characteristics of the ionospheric Es layer clutter basically, and the JDL algorithm can suppress ionospheric Es layer clutter well. This method provided a theoretical basis for the ionospheric clutter suppression technology and the use of STAP in anti-ionospheric clutter.

\section{Acknowledgments}

At the point of finishing this paper, one of the authors would like to express his/her sincere thanks to the National Natural Science Foundation Key Project (no. 61032011) and members of the School of Electronics and Information Engineering, Research Center, Harbin Institute of Technology for technical support.

\section{References}

[1] H. C. Chan, "Characterization of ionospheric clutter in HF surface wave radar," Tech. Rep. 2003-114, Defence R\&D Canada, Ottawa, Canada, 2003.

[2] D. L. Knepp, "Multiple phase-screen calculation of the temporal behavior of stochastic waves," Proceedings of the IEEE, vol. 71, no. 6, pp. 722-737, 1983.

[3] Y. W. Kiang and C. H. Liu, "Multiple phase-screen simulation of HF wave propagation in the turbulent stratified ionosphere," Radio Science, vol. 20, no. 3, pp. 652-668, 1985.

[4] J. F. Wagen and K. C. Yeh, "A numerical study of waves reflected from a turbulent ionosphere," Radio Science, vol. 21, no. 4, pp. 538-604, 1986.
[5] D. L. Knepp and L. J. Nickisch, "Multiple phase screen calculation of wide bandwidth propagation," Radio Science, vol. 44, p. $1,2009$.

[6] M. Ravan, R. J. Riddolls, and R. S. Adve, "Ionospheric and auroral clutter models for HF surface wave and over-thehorizon radar systems," Radio Science, vol. 47, no. 3, 2012.

[7] F. J. Massey, "The Kolmogorov-Smirnov test for goodness of fit," Journal of the American Statistical Association, vol. 46, no. 253, pp. 68-78, 1951.

[8] M. W. Y. Poon, R. H. Khan, and S. Le-Ngoc, "Singular value decomposition (SVD) based method for suppressing ocean clutter in high frequency radar," IEEE Transactions on Signal Processing, vol. 41, no. 3, pp. 1421-1425, 1993.

[9] M. Ravan, O. Saleh, and R. Adve, "KB-STAP implementaion for HFSWR: final report," Tech. Rep. W7713-060999/001/SV, 2008.

[10] O. Saleh, Adaptive processing in HF surface wave radar [M.S. thesis], University of Toronto, 2008.

[11] O. Saleh, R. S. Adve, R. J. Riddolls, M. Ravan, and K. Plataniotis, "Adaptive processing in high frequency surface wave radar," in Proceedings of the IEEE Radar Conference, RADAR 2008, pp. 16, Rome, Italy, May 2008.

[12] G. A. Fabrizio and A. Farina, "GLRT-based adaptive doppler processing for HF radar systems," in Proceedings of the IEEE International Conference on Acoustics, Speech and Signal Processing (ICASSP '07), pp. II949-II952, Honolulu, Hawaii, USA, April 2007.

[13] E. J. Yang, J. Chun, R. Adve, and J. Chun, "A hybrid D3sigma delta STAP algorithm in non-homogeneous clutter," in Proceedings of the IET International Conference on Radar Systems (RADAR '07), p. 76, Edinburgh, UK, October 2007.

[14] E. Aboutanios and B. Mulgrew, "Hybrid detection approach for STAP in heterogeneous clutter," IEEE Transactions on Aerospace and Electronic Systems, vol. 46, no. 3, pp. 1021-1033, 2010.

[15] J. Goldstein, I. Reed, and P. Zulch, "Multistage partially adaptive STAP CFAR detection algorithm," IEEE Transactions on Aerospace and Electronic Systems, vol. 35, no. 2, pp. 645-651, 1999. 

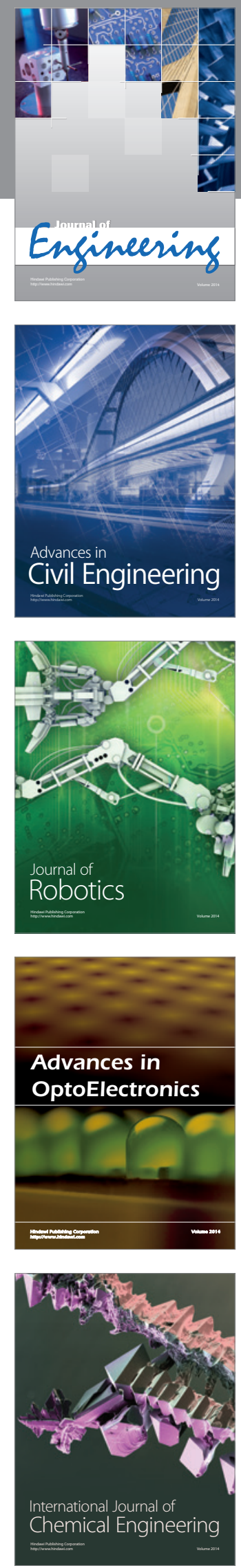

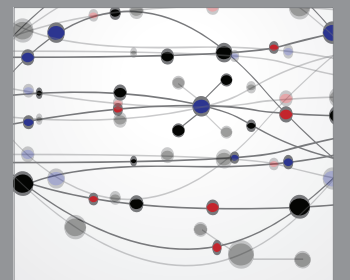

The Scientific World Journal
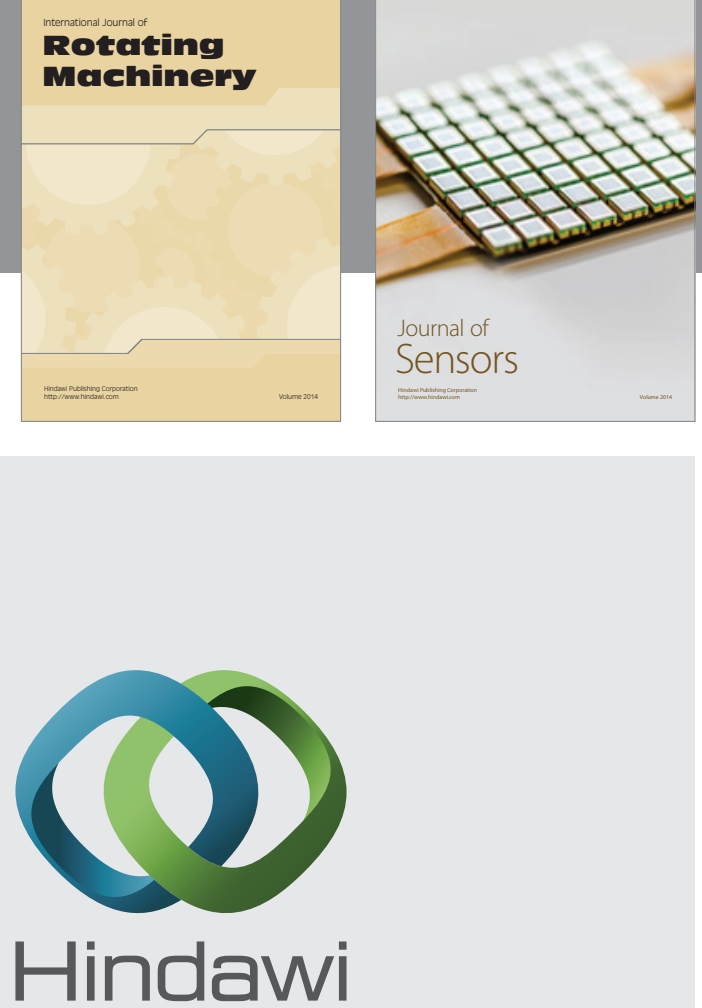

Submit your manuscripts at http://www.hindawi.com
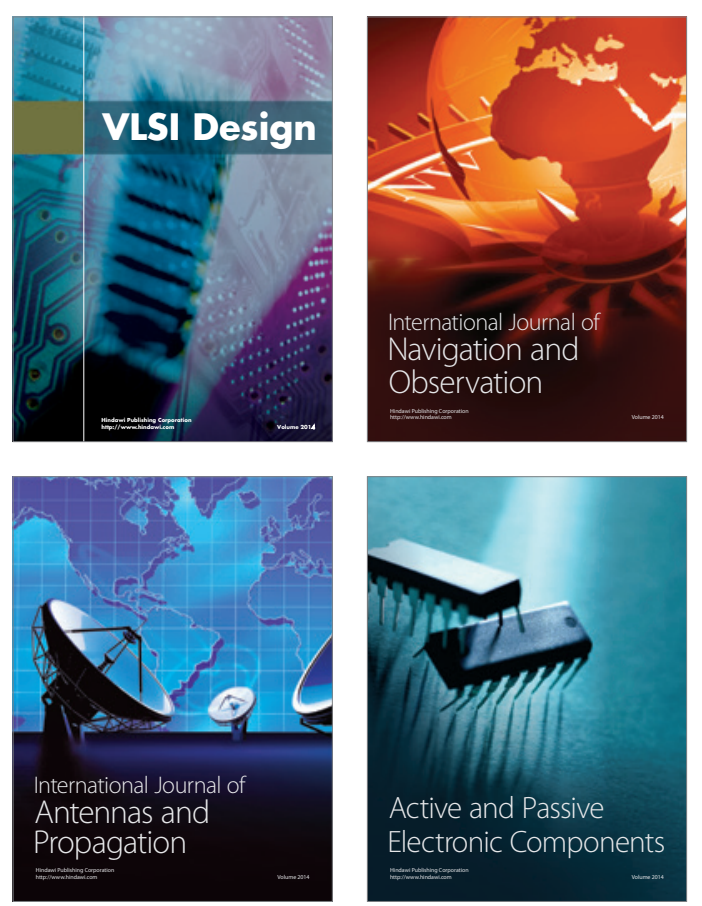
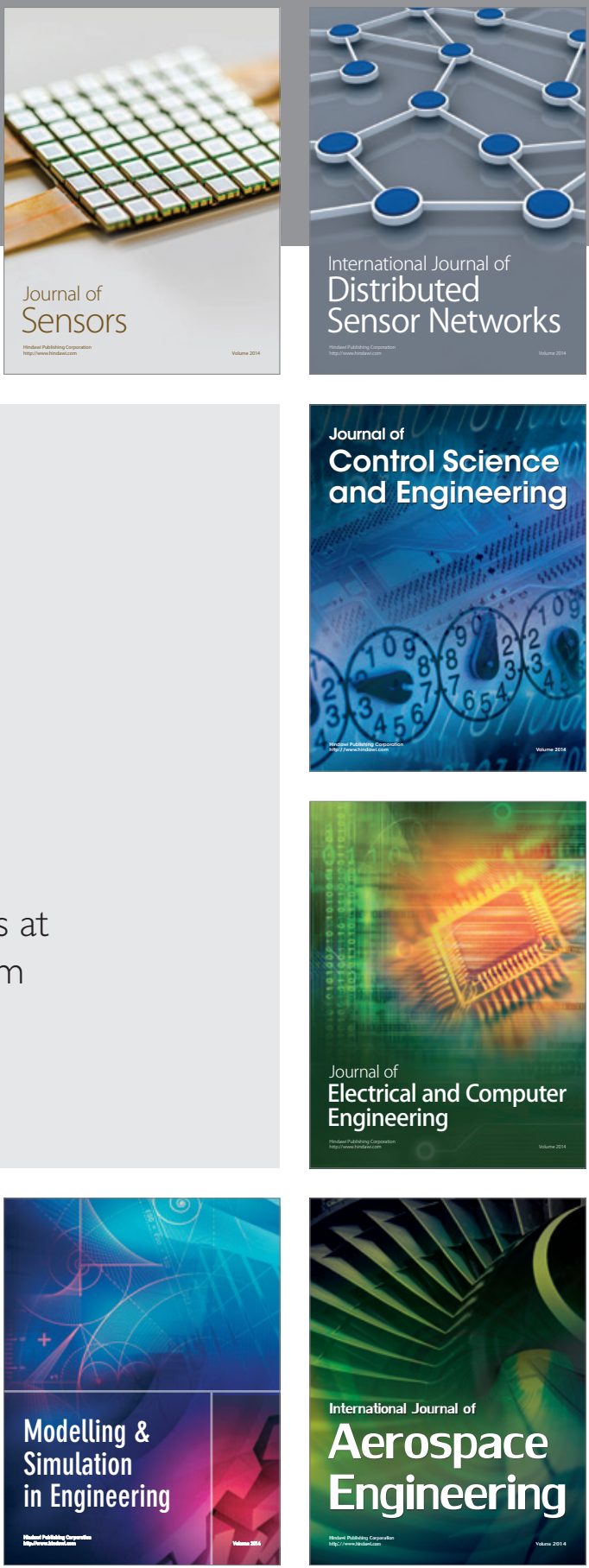

Journal of

Control Science

and Engineering
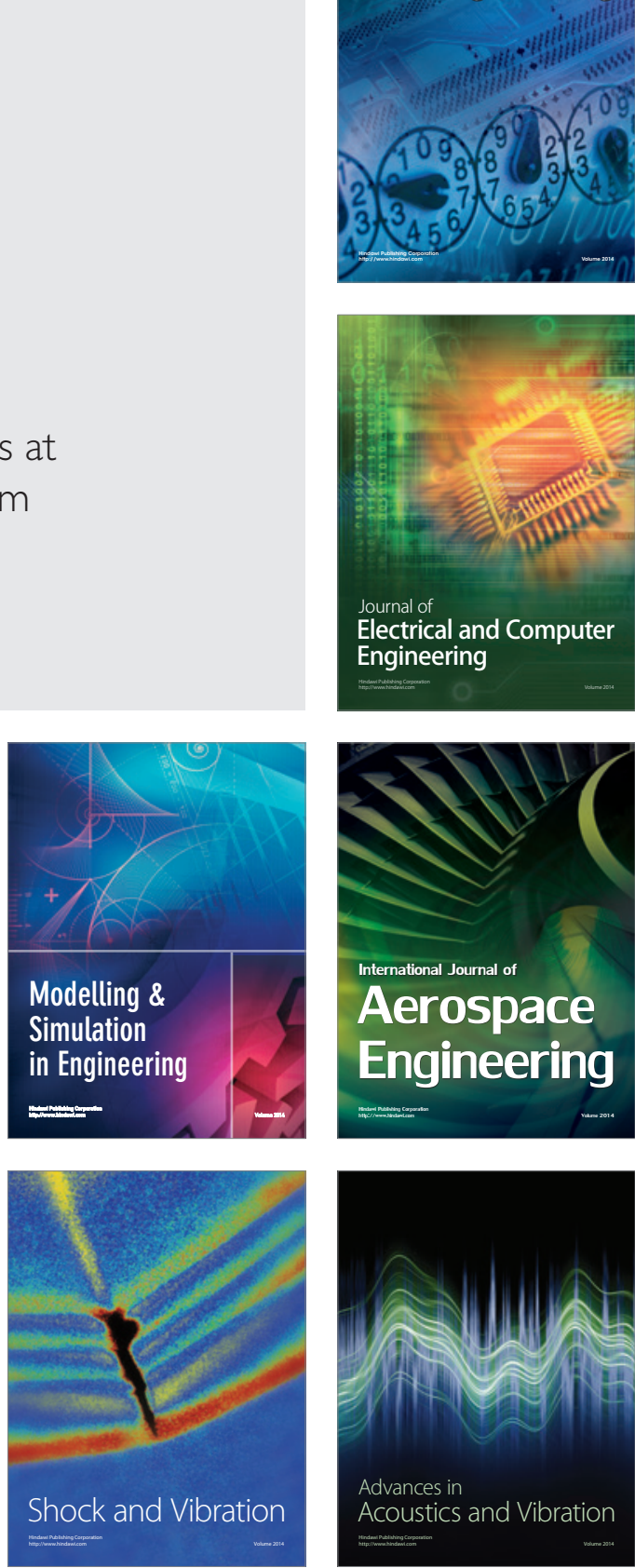